\section{ABSTRACT}

\title{
Transcription-coupled structural dynamics of topologically associating domains regulate replication origin efficiency
} Yongzheng $\mathrm{Li}^{1 \#}$, Boxin Xue ${ }^{1 \#}$, Liwei Zhang ${ }^{2}$, Qian Peter Su${ }^{1,3}$, Mengling Zhang ${ }^{1}$, Haizhen Long ${ }^{2}$, Yao Wang ${ }^{1}$, Yanyan $\mathrm{Jin}^{4}$, Yingping $\mathrm{Hou}^{1}$, Yuan $\mathrm{Cao}^{1}$, Guohong $\mathrm{Li}^{2}, 5$, Yujie Sun $^{1 *}$

${ }^{I}$ State Key Laboratory of Membrane Biology, Biomedical Pioneer Innovation Center (BIOPIC), School of Life Sciences, Peking University, Beijing, China 100871

${ }^{2}$ National Laboratory of Biomacromolecules, CAS Center for Excellence in Biomacromolecules, Institute of Biophysics, Chinese Academy of Sciences, Beijing, China, 100101

${ }^{3}$ Institute for Biomedical Materials \& Devices (IBMD), Faculty of Science, the University of Technology Sydney, Ultimo, NSW 2007, Australia.

${ }^{4}$ Department of Neurobiology, Beijing Centre of Neural Regeneration and Repair, Capital Medical University, Beijing, China 100101

${ }^{5}$ University of Chinese Academy of Sciences, Beijing, China, 100049

\# These authors contribute to the work equally.

*Email: sun_yujie@pku.edu.cn

Metazoan cells only utilize a small subset of the potential DNA replication origins to duplicate the whole genome in each cell cycle. Origin choice is linked to cell growth, differentiation, and replication stress. Despite various genetic and epigenetic signatures are found to be related with active origins, it remains elusive how the selection of origins is determined. The classic Rosette model proposes that the origins clustered in a chromatin domain are preferentially and simultaneously fired, but direct imaging evidence has been lacking due to insufficient spatial resolution. Here, we applied dual-color stochastic optical reconstruction microscopy (STORM) super-resolution imaging to map the spatial distribution of origins within individual topologically associating domains (TADs). We found that multiple replication origins initiate separately at the spatial boundary of a TAD at the beginning of the S phase, in contrary to the Rosette model. Intriguingly, while both active and dormant origins are distributed homogeneously in the TAD during the G1 phase, active origins relocate to the TAD periphery before entering the $\mathrm{S}$ phase. We proved that such origin relocalization is dependent on both transcription and CTCF-mediated chromatin structure. Further, we observed that the replication machinery protein PCNA forms immobile clusters around the TADs at the G1/S transition, which explains why origins at the TAD periphery are preferentially fired. Thus, we propose a "Chromatin Re-organization Induced Selective Initiation" (CRISI) model that the transcriptioncoupled chromatin structural re-organization determines the selection of replication origins, which transcends the scope of specific genetic and epigenetic signatures for origin efficiency. Our in situ super-resolution imaging unveiled coordination among DNA replication, transcription, and chromatin organization inside individual TADs, providing new insights into the biological functions of sub-domain chromatin structural dynamics. 
42 KEY WORDS: replication origin, topologically associating domain (TAD), chromatin 43 structure, transcription, super-resolution imaging, STORM.

\section{INTRODUCTION}

DNA replication is an exquisitely regulated process. Its deregulation may lead to genome

47 instability and tumorigenesis [1]. In metazoans, duplication of the genome is initiated at tens of 48 thousands of discrete chromosome loci known as replication origins. Intriguingly, while a 49 mammalian cell has a total of $\sim 250,000$ potential replication origins, it only uses a small subset $50(\sim 10 \%)$ to duplicate the whole genome [2-5]. Dormant origins can be initiated to rescue stalled 51 replication forks, facilitating accurate and timely genome replication under stress $[6,7]$.

52 Whether the selection of origins is random or regulated has been under debate. Single 53 cell-based measurements, including the classic DNA combing assays [8-10] and the recent 54 single-cell sequencing studies [11, 12], showed that cells rarely use the same cohort of origins 55 to duplicate the genome. Nevertheless, either single cell or population-averaged origin mapping 56 experiments have confirmed that not all origins are equal, and they rather have differential 57 probabilities of firing, namely origin efficiency [4], against a random origin selection 58 mechanism.

59 The mechanisms that determine the origin efficiency remain enigmatic. Various genetic 60 and epigenetic signatures, including $\mathrm{CpG}$ islands, G-quadruplexes, nucleosome-depleted 61 regions, and histone modifications, are found to correlate with origin efficiency, but a consensus 62 principle is still lacking [2]. The origin efficiency has been also suggested to link with chromatin 63 structures $[4,13,14]$. Several earlier studies have revealed a relationship between replicons and 64 chromatin loops [9, 15-17]. Beyond the loop structure, more recent studies have shown that the 65 spatiotemporal initiation of replication is regulated at the chromatin domain level. Genome66 scale mapping of replication kinetics showed that DNA replication in metazoan cells takes place 67 in a defined temporal order with the genome segmented into large chromosomal regions, known 68 as replication domains (RDs) [4, 5]. Each RD contains multiple replicons with uniform and 69 constant replication timing. Importantly, the boundaries of RDs are found to align precisely 70 with that of topologically associating domains (TADs) [18]. TADs are physical 71 compartmentalization units of the genome that are stable over multiple cell cycles and 72 conserved across related species [19]. Thus, this finding provides strong supports for the 73 correlation between DNA replication and the three-dimensional (3D) structure of chromosomes. 74 A typical $\mathrm{RD}$ is about $1 \mathrm{Mb}$ and contains a few dozens of potential origins. These origins do 75 not have similar replication efficiencies as only several of them are actually used to replicate 76 the domain [2-5]. In temporal space, direct measurements on spread-out DNA fibers by DNA 77 combing experiments have shown that the active origins within a RD fire nearly synchronously 78 [8-10]. However, how the origin efficiency is spatially regulated in a RD has been an 
outstanding question.

In physical space, mapping of the spatial arrangements of replication sites by in situ fluorescence imaging in the nucleus showed that DNA replication initiates at thousands discrete puncta termed replication foci (RFi) [9, 20-25]. Provided that the number of pulse-labelled RFi is much less than the number of active origins [9, 20-22], RFi are thought to be the equivalents of RDs defined in temporal space and contain multiple replicons. Based on the collective evidence from the DNA halo [9, 15-17], DNA combing [8, 9, 26] and RFi imaging [9, 20-22], the Rosette model was proposed to illustrate the spatiotemporal organization and regulation of active origins in a $\mathrm{RD}$ [4]. In this model, a $\mathrm{RD}$ contains multiple loops which form a rosettelike structure with the active origins clustered and co-fired in the chromatin domain. The Rosette model is further supported by another study showing that depletion of cohesin, a protein complex scaffolding the rosette-like structure, reduces the number of origins used for genome duplication [27]. However, as previous imaging studies were mostly limited in their spatial resolution and lack of sequence-specific labeling of TADs and replication origins, there is no direct imaging evidence to support the clustered origin distribution. In a recent work, Cardoso and his colleagues applied SIM super-resolution imaging (resolution $\sim 100 \mathrm{~nm}$ ) and identified more replicons than conventional imaging (resolution $\sim 300 \mathrm{~nm}$ ) [23]. Importantly, with the improvement in resolution, nearly all replicons are found to be spatially separated at the beginning of the $\mathrm{S}$ phase, which casts doubt on the clustering of replication origins proposed in the Rosette model. In the accompanying work, they proposed a stochastic, proximity-induced (domino-like) replication initiation model, in which the active origins are not necessarily clustered spatially in the domain but the domino-like replication progression leads to clustering of replicons [28].

A thorough understanding of how the physical structure of RDs regulates origin efficiency needs in situ imaging of the spatial distribution of both active and dormant origins within the TADs. Given that a TAD is about $800 \mathrm{~kb}$ [18] with a radius of gyration less than $300 \mathrm{~nm}$ [29] and contains a few dozens of potential origins, dual-color 3D super-resolution imaging with ultra-high resolution in all three dimensions is a pre-requisite to distinguish which origins are more preferentially used among the many potential candidates within individual TADs. Moreover, new labelling strategies are necessary for dormant origins because the traditional approach based on metabolic pulse-labeling only labels active origins. Here, we applied a recently developed chromatin painting and imaging technique, namely OligoSTORM [30, 31], to investigate whether origin efficiency is dependent on TAD structure. OligoSTORM combines Oligopaints [32] with stochastic optical reconstruction microscopy (STORM) [33].

3 Oligopaints are highly efficient oligonucleotide FISH (fluorescent in situ hybridization) probes 4 based on PCR strategy that can robustly label whole chromosomes or any specific chromosomal 5 regions. STORM and its equivalents PALM/fPALM [34, 35] are single-molecule localization- 
based super-resolution imaging techniques that have the highest spatial resolution $(\sim 20 \mathrm{~nm}$ laterally and $\sim 50 \mathrm{~nm}$ axially) among all super-resolution imaging methods [36]. With the best of both sides, OligoSTORM has been successfully applied to resolve the fine physical chromatin structures, such as TADs and compartments, in single cells [29, 37, 38].

Using OligoSTORM, we performed, to our knowledge, the first quantitative characterization of TAD structural dynamics and the spatiotemporal distribution of replication origins within individual TADs in different cell cycle stages at sub-diffraction-limit resolution. We discovered that in the G1 phase, TADs undergo a transcription-dependent structural reorganization process, which exposes active origins to the spatial boundary of the TAD; in contrast, dormant origins remain inside the TAD. We also observed an interesting peri-RFi distribution of the major replication machinery protein PCNA, in line with the observation that replication initiation generally takes place at the spatial boundary of a TAD. Thus, our work reveals a new origin selection mechanism that the replication efficiency of origins is determined by their physical distribution in the chromatin domain and transcription plays a role in the chromatin structural re-organization. This new mechanism transcends the scope of specific genetic and epigenetic signatures for origin efficiency and also provides new insight into the biological functions of sub-domain chromatin structural dynamics.

\section{RESULTS}

\section{Replication origins initiate separately at the periphery of a TAD}

In order to investigate the role of chromatin structure in origin selection, we chose to directly visualize how replication initiation is spatially organized and regulated within individual RDs using STORM imaging. Two RDs were chosen from the replication timing profile of HeLa cells (Appendix Figure S1a). The boundaries of either RD are overlaid with that of a TAD, which are hereafter named as TAD1 and TAD2, respectively (Appendix Figure S1b). TAD1 (Chr1:16911932-17714928) is an early replicating domain and enriched of transcriptionally active histone modifications. TAD2 (Chr1: 17722716-18846245) is a middle replicating domain and enriched of transcriptionally repressed histone modifications (Appendix Figure S1c). The two TADs were labeled by the Oligopaint approach using 12,000 TAMRAmodified primary oligonucleotide probes targeting the TADs and then imaged by STORM (Methods). Morphological characterization showed that the radii of gyration of TAD1 and TAD2 are about $200 \mathrm{~nm}$ (Appendix Figure S2), which is consistent with previous work [29, 38]. Moreover, even though the genomic length of TAD2 is larger than that of TAD1, the physical size of TAD2 is significantly smaller than that of TAD1 (Appendix Figure S2b), suggesting that TAD2 is more compact. This observation is consistent with the previously

1 reported findings that active chromatin domains are more open than repressed chromatin 2 domains [29, 38], thereby benchmarking the technical rigor of our TAD labeling and imaging. 
Next, to visualize the replication initiation sites in the TADs, we took the classic metabolic 154 pulse-labeling strategy [9]. Briefly, we first synchronized HeLa cells to the boundary of the G1 155 phase and S phase as previously described [9, 39] (Methods). Immediately after release of 156 aphidicolin arrest at the G1/S boundary, we performed a 10-min pulse labeling of the replicating 157 DNA by supplying thymidine analog EdU, which was then labeled with Alexa647 by click 158 chemistry after fixation of the cell (Methods). This synchronization procedure can synchronize 159 nearly $80 \%$ cells at the G1/S transition and minimally impacts the growth and morphology of 160 cells [29, 40] (Appendix Figure S3). Following labeling and fixation, we applied dual-color 161 STORM (Methods) to image the TADs (Figure 1a, green) and the replication initiation sites, 162 which appeared as punctate foci (Figure 1a, purple). The punctate distribution of 10-min 163 pulse labeled foci imaged by STORM in our work were similar with those were previously 164 imaged by other groups with SIM or STORM [23]. The positions of these foci can precisely 165 represent the position of the corresponding replication initiation sites for two reasons. First, as 166 EdU was added immediately after the release of replication arrest, majority of the pulse-labeled 167 RFi were supposed to contain the corresponding replication initiation sites. Second, although 168 the sequence length of the DNA replicated over a 10-min period was quite long (roughly $20 \mathrm{~kb}$ $169[9,23,40])$, its physical size was only $30 \mathrm{~nm}$ in diameter, as revealed in the super-resolution 170 images (Figure 1a, Appendix Figure S6c). This diameter is close to the lateral resolution of 171 STORM imaging. As the spatial resolution (directly related with the single-molecule 172 localization precision) sets the minimal apparent size of a target imaged by STORM [33], there 173 would be no difference in the apparent size or position when imaging a $20 \mathrm{~kb}$ genomic region 174 and a much smaller sub-region, e.g., the replication initiation site in the region.

Intriguingly, the replication initiation sites seemed to preferentially localize at the physical 176 boundary of the early replicating TAD1 as shown in the two insets in the upper left panel of 177 Figure 1a, which are close-up view of the two allelic TAD1 and their associated replication 178 initiation sites. We adopted a recently developed robust and unbiased segmentation algorithm, 179 SR-Tesseler [41], to quantitatively analyze TADs and origins in super-resolution images 180 (Methods \& Appendix Figure S4). To describe the relative spatial relationship, we defined 181 barycenter distance as the physical distance between the barycenter of a TAD and the barycenter 182 of RFi normalized by the radius of gyration of the TAD (Figure 1b) (Methods). The barycenter 183 is the mass density center of all single molecule localizations in a TAD or RFi. For randomly 184 distributed foci within the TAD, the expected mean barycenter distance is 0.71 (Methods). In 185 contrast, the barycenter distances between the 10-min pulse-labeled RFi and TAD1 were near 1861 (Figure 1b), indicating a peripheral distribution of the replication initiation sites relative to 187 TAD1. Importantly, the barycenter distance of RFi labeled for $15 \mathrm{~min}$ were closer to the center 188 of TAD1 in comparison with RFi labeled for 10 min (Figure 1b), showing that our method is 
highly sensitive as a means of detecting the spatial distribution of replication origins in a TAD. Moreover, RFi labeled for 60 min were well overlaid on TAD1 (Figure 1a \& Appendix Figure S5b) with barycenter distances near 0.5 (Figure 1b \& Appendix Figure S5). As a control, RFi labeled for 1 hour starting at the G1/S boundary did not show obvious overlap with the middle replicating TAD2 (Figure 1a, b), consistent with the fact that TAD2 begins to replicate at approximately 3 hours into the S phase (Appendix Figure S1 \& Figure 2a).

To further validate the analysis of spatial localization of RFi relative to the TAD, we also applied DBSCAN [42], a density-based spatial clustering algorithm, to extract individual RFi and quantify the spatial localization of RFi in TADs in 2D and 3D STORM images (Appendix Figure S5) (Methods). The spatial relationship of RFi relative to the TAD (Appendix Figure S5a-c) rendered by DBSCAN in 2D or 3D images (Appendix Figure S5a-c) was identical with those obtained by the SR-Tesseler analysis (Figure 1b). We also defined the radial density distribution (RDD), which is the median radial distribution of all single-molecule detections of the RFi in a TAD normalized by the radius of the TAD, to quantify the spatial localization of RFi in TADs in 3D images (Methods). A larger RDD value indicates a more peripheral distribution of RFi in a TAD. The spatial distribution of RFi revealed by the RDD analysis was similar to that obtained by the barycenter distance analysis (Appendix Figure S5c, d). The analyses described above cross validated each other and eliminated the possibility of artifacts introduced by foci identification, inter-foci distance measurement algorithms, or projection of $3 \mathrm{D}$ images onto the $2 \mathrm{D}$ plane. Therefore, these data demonstrated that multiple replication origins initiate separately at the spatial periphery of TAD1.

Next, to check whether the above findings obtained with TAD1 are generally true for all early replicating TADs, a high-throughput labeling method is needed. Provided that the boundaries of RDs and TADs are precisely aligned [18], we took a metabolic labeling strategy to label all early RDs and their associated replication initiation sites by two rounds of BrdU and EdU pulse labeling at the beginning of the $\mathrm{S}$ phase over two consecutive cell cycles, respectively (Figure 1c). EdU was labeled with Alexa647 by click chemistry, whereas BrdU was immunostained with atto-550 [9]. It has been suggested that RFi labeled by thymidine analogs EdU or BrdU for 1 hour generally correspond to RDs [9], as replication of early RDs takes approximately 1 hour $[9,21,43,44]$. This assumption was validated by large overlapping between FISH-labeled TAD1 and its corresponding 60-min EdU-labeled RFi (Figure 1a lower left, Appendix Figure S5, Appendix Figure S6a, b). Because the spatial density of 60-min metabolically labeled RFi was very high, which impeded the confidence in the algorithm with regard to identification of the boundaries between spatially adjacent RDs, we chose to label RDs using 45-min labeling duration. As shown by the dual-color STORM imaging in Figure 1c, early RDs double-labeled for $45 \mathrm{~min}$ and $60 \mathrm{~min}$ in two consecutive cell cycles merged very 
$22645 \mathrm{~min}$ were slightly smaller than those labeled for $60 \mathrm{~min}$, albeit insignificant (Appendix 227 Figure S6c), supporting the usage of 45-min labeled RFi to represent early RDs. As a control 228 experiment, we showed that the size increase from RFi labeled for 10 min to those labeled for 22915 min was successfully detected (Appendix Figure S6c), demonstrating the high detection 230 sensitivity of STORM imaging and also excluding the possibility that the insignificant size 231 difference between RFi labeled for $45 \mathrm{~min}$ and $60 \mathrm{~min}$ was due to insufficient detection 232 sensitivity. We also checked whether different thymidine analogs, e.g. EdU and BrdU, 233 introduced any difference in the size of RFi. The STORM images showed no significant difference between EdU- and BrdU-labeled RFi (Appendix Figure S6d).

We then labeled all early RDs with 45-min BrdU in the first cell cycle followed by labeling 236 of the replication initiation sites with 10-min EdU in the second cell cycle (Figure 1c right). We 237 obtained a global view of the spatial distribution of replication initiation sites relative to early 238 RDs in a cell. Analysis of the dual-color STORM images showed that there were averagely 7 239 replication initiation sites in each $\mathrm{RD}$, in good agreement with previous estimations $[5,45]$ as 240 well as the fact that the sizes of a RD and a replicon are about $800 \mathrm{~kb}$ and $120 \mathrm{~kb}$, respectively.

241 These analyses thereby benchmarked the technical rigor of labeling and imaging of RDs and associated origins.

We next calculated the barycenter distances between replication initiation sites (EdU 10 $244 \mathrm{~min}$ ) and RDs (BrdU $45 \mathrm{~min}$ ) and found that they were significantly larger than those between 245 doubly labeled (EdU 60 min and BrdU 45 min) RDs (Figure 1d). Lastly, a similar spatial pattern 246 was observed when Cy3B or dUTP was used respectively instead of atto-550 or BrdU, thereby

247 eliminating the possibility that the observed pattern could be the consequence of labeling or detection artifacts associated with specific dyes (Appendix Figure S7). Taken together the data of both particular RDs and metabolically-labeled RDs, we conclude the fired replication origins in a RD are spatially separated, which is in direct contrary with the classic model [4] and in line with recent findings discovered by super-resolution imaging [23]. More importantly, these spatially separated replication origins tend to initiate at the periphery of RDs, implicating a role of chromatin domain structure in regulating the efficiency of replication origins.

\section{Active origins relocate inside-out to the periphery of early replicating TADs in the G1} phase

Only $10-20 \%$ of the origins in a TAD are used for DNA replication during each cell cycle, while the rest stay dormant. Given the observation that replication tends to initiate at the periphery of an early replicating TAD (Figure $1 \&$ Appendix Figure S5), we next set to image both active and dormant origins to check whether the spatial distribution of origins in a TAD is related to their replication efficiency. As dormant origins cannot be marked with metabolic 
263 pulse labeling, OligoSTORM was applied to label and image the TADs and origins. To obtain

264 the replication efficiency of origins in TAD1 and TAD2, we first measured the dynamic 265 replication profile of HeLa cells using BrdU-seq [46] by 10-min BrdU labeling at 0 min, 1 hour, 2663 hours and 6 hours into the S phase (Figure 2a, black peaks). The BrdU-seq profile reveals that 267 TAD1 replicates in the first hour of the S phase, while TAD2 starts to replicate after about 3 268 hours, which is in line with the replication timing profile (Appendix Figure S1). All potential 269 replication origins in TAD1 and TAD2 were mapped by ORC1 and H2A.Z ChIP-seq [47] of $270 \mathrm{HeLa}$ cells. We aligned the dynamic replication profile and ORC1 binding sites with the 271 previously reported replication landscape of the HeLa cell genome [48] (Figure 2a). Based on 272 the origin efficiency revealed by both the replication landscape and the dynamic replication 273 profile, 3 representative active origins (ORI1, ORI2 and ORI3, marked by yellow and red boxes) 274 and 2 representative dormant origins (ORI4 and ORI5, marked by black boxes) were chosen in 275 TAD1. Two active origins (ORI6 and ORI7, marked by red boxes) were chosen in TAD2 276 (Additional File Table 1 for detailed information of TADs and origins).

277 We applied dual-color OligoSTORM to image the TAD and its associated origins at the $278 \mathrm{G} 1 / \mathrm{S}$ transition without releasing aphidicolin arrest (Methods). In order to ensure that the target 279 replication origin was labeled with ample fluorescent signal, Oligopaint probes were designed 280 to target a $\sim 20 \mathrm{~kb}$ genomic zone containing the replication origin (Methods). The results showed 281 that at the G1/S transition, all 3 active origins in TAD1 were located at the spatial periphery of 282 TAD1 (Figure 2b, upper) with large barycenter distances (Figure 2c, red). In contrast to the 283 active origins, the dormant origins (ORI4 and ORI5) tended to locate at the interior of TAD1 284 (Figure 2b, upper) with barycenter distances much shorter than those of ORI1, ORI2 and ORI3 285 (Figure 2c, red). Interestingly, the active origins in TAD2 (ORI6 and ORI7), which are not 286 supposed to fire until the middle $\mathrm{S}$ phase, tended to locate inside of the domain at the G1/S 287 transition (Figure 2b, upper) with small barycenter distances (Figure. 2c, red). Taken together, 288 these results suggest that the replication efficiency of origins at the G1/S transition is correlated 289 with their physical positions in the TAD.

290 Eukaryotic DNA replication is tightly orchestrated with the cell cycle. In the canonical 291 two-step activation model [4], licensing of origins occurs with pre-RC formation in the G1 292 phase followed by origin activation and initiation in the S phase. Recent Hi-C studies have 293 shown that the structure of TADs changes from the G1 phase to the S phase [49]. We wondered 294 how the spatial distribution of origins in a TAD changes accompanying the chromatin structure, 295 which could serve as determinants of selective origin initiation. We thus imaged the TAD and 296 origins in the mid-G1 phase (approximately 5 hours post G1 onset) (Methods), which is after 297 the timing decision point (TDP) when the replication timing program becomes established and 298 TADs reform [50]. Strikingly, we found that the active origins ORI2 and ORI3 were located 299 inside of TAD1 in the mid-G1 phase (Figure 2b, lower) with small barycenter distances (Figure 
300

301

302

303

304

305

306

307

308

309

310

311

312

2c, blue), in sharp contrast to their peripheral localization in TAD1 at the G1/S transition (Figure 2b, upper; Figure 2c, red). On the contrary, dormant origins ORI4 and ORI5 were found to remain inside of TAD1 from the mid-G1 (Figure 2b, lower; Figure 2c, blue) to the G1/S transition (Figure 2b, upper; Figure 2c, red). These observations suggested that active origins undergo an inside-out relocation process in the TAD, possibly along with the chromatin structural re-organization within the TADs that occurs in the G1 phase. Interestingly, unlike ORI2 and ORI3, active origin ORI1 did not relocate but remained at the TAD periphery from the mid-G1 phase to the G1/S transition (Figure 2b, c). We note that, in the sequence space, ORI1 is at the insulation boundary of TAD1 (Figure 2a and Additional File Table 1), and therefore structural re-organization within the TADs would not affect its peripheral localization relative to the TAD. Such correspondence between the sequence boundary and the physical boundary of a TAD was also reported in a recent study [38], thereby again benchmarking the technical rigor of labeling and imaging of TADs and associated origins.

To further investigate the relationship of replication origins and chromatin loops within the TADs, we aligned origins with the sites enriched of CTCF and cohesin genome wide (Methods) (Appendix Figure S8). CTCF and cohesin are the key scaffold protein complexes bound at the anchor sites of the chromatin loops as well as the TAD boundary [51]. In general, compared with random DNA loci, CTCF-cohesin binding sites were enriched with replication origins. Active origins co-localized better with CTCF-cohesin binding sites than dormant origins. In addition, active origins located at TAD boundaries (Methods) were of higher replication efficiency than those located inside the TADs. The sequencing results again emphasized the relationship of replication efficiency with chromatin organization within the TADs.

In addition to the structural re-organization within the TADs, we found that the physical size of TAD1 also became larger at the G1/S transition in comparison with its size in the G1 phase (Figure 2d), while this change was not detected for TAD2. Note that the volume increase was not the result of DNA replication, as the cells were arrested at the G1/S transition, suggesting that the chromatin of TAD1 undergoes de-compaction in the G1 phase, which is in line with the results of $\mathrm{Hi}-\mathrm{C}$ analysis showing that intra-domain chromatin interactions decrease in the G1 phase [49]. Analysis of 3D STORM images led to the same findings (Appendix Figure S9), which again eliminated the possibility of artifacts introduced by projection of 3D images onto the 2D plane. Taken together, these data revealed that the structural re-organization within the TADs and decompaction in the G1 phase facilitate the relocation of active origins from the TAD interior to the periphery, supporting the observation that DNA replication initiates at the periphery of TADs in the beginning of S phase (Figure 1). 
337 Distinct spatial localization of active and dormant origins at the G1/S transition is 338 correlated with chromatin loops and dependent on transcription

339 Next, we set to explore the factors that are responsible for differential origin localization in 340 the TAD. We first examined the effects of CTCF and cohesin, the key scaffold protein complexes responsible for loop formation in a TAD [51]. Upon down-regulation of CTCF or cohesin using RNAi (Figure 3a, insets), we found that the active origins (ORI2 and ORI3) was not relocated to the TAD periphery at the G1/S transition in both 2D and 3D images (Figure 3a

344 \& Appendix Figure S10a). More importantly, the barycenter distances of either active origins or dormant origins relative to TAD1 became similar with that of randomly distributed foci (about 0.7) (Figure 3b \& Appendix Figure S10b), indicating disappearance of differential origin distribution. Such effect was likely due to the scrambling of chromatin structure within the TADs upon loss of CTCF or cohesin, which is in line with the Hi-C data that depletion of either cohesin or CTCF eliminates loops $[52,53]$. These results suggested that the relocation of replication origins in the G1 phase is dependent on chromatin looping mediated by CTCF and cohesin in the TAD.

Previous studies have shown that in the G1 phase, transcription activity is generally high in early RDs and active origins abut actively transcribed genes [48, 54]. Indeed, expression of genes associated with active replication origins (ORI1, ORI2, ORI3) in TAD1 is higher than that for dormant replication origins (ORI4, ORI5) (Appendix Figure S11). Transcription has also been found to fundamentally influence chromatin structures at different levels and through various mechanisms, including nucleosome disassembly, enhancer-promoter loop formation, transcript cis-interaction, CTCF and cohesin displacement, gene relocation and transcription factory formation [55-57]. Moreover, in a recent study, Gilbert and his colleagues identified cis-acting elements, namely early replicating control elements (ERCEs), which regulate the 361 replication timing and the structure of TADs [58]. Importantly, ERCEs have properties of enhancers or promoters, implicating a fundamental role of transcription in orchestrating genome replication and chromatin architecture. Therefore, given that the origin relocation takes place in the G1 phase, we next examined whether transcription in the G1 phase contributes to chromatin structural re-organization within the TADs. To do so, we treated cells with transcription elongation inhibitor 5,6-dichloro-1- $\beta$-D-ribofuranosyl-benzimidazole (DRB) [59] from the mid-G1 phase to the G1/S transition, after which we labeled TAD1 and its replication

368 origins using Oligopaint probes. Interestingly, upon transcription inhibition by DRB treatment, 369 active origins ORI2 and ORI3 were no longer found to relocate to the periphery of the TAD at 370 the G1/S transition in both 2D and 3D images (Figure 3c \& Appendix Figure S10c) and had 371 barycenter distances similar to those of dormant origins (Figure 3d \& Appendix Figure S10d).

372 Moreover, the radius of TAD1 at the G1/S transition in DRB-treated cells was found to be 373 smaller than that in normal cells (Figure 3e \& Appendix Figure S10e) and similar with that 
observed in the G1 phase (Figure 2d \& Appendix Figure S9c). This observation suggested that transcription de-compacts the chromatin structure of TADs. Taken together, these results demonstrated that transcription-dependent chromatin structural re-organization within the TADs exposes a subset of origins to the physical boundary of a TAD, which are preferentially used for replication initiation.

\section{Replication elongation factor PCNA surrounds TADs both in the G1 and G1/S phases.}

To answer why origins located on the physical boundary of a TAD are preferentially used for DNA replication, we examined the spatial distribution of replication machinery relative to individual TADs at the G1 phase and G1/S phases by imaging proliferating cell nuclear antigen (PCNA) [60]. As a control, we also monitored the distribution of minichromosome maintenance complex component 2 (MCM2) and CTCF, respectively. Provided that metabolically labeled RDs merge well with FISH-labeled TADs in the early S phase (Figure 1, Appendix Figure S5 and Appendix Figure S6), to label early replicating TADs and protein factors in the same cell, TADs were first labeled by supplying cells with EdU for 45 mins immediately after release of aphidicolin arrest at the beginning of the S phase; in the next cell cycle, the cells were fixed and immunostained at either the mid-G1 or G1/S phase. The EdU-labeled TADs became larger from the mid-G1 phase to the G1/S transition (Appendix Figure S12), in line with the observation of FISH-labeled TADs (Fig. 2d). As a scaffold factor of TADs, CTCF formed large foci (Figure 4a) and neither their distribution relative to the TADs (Figure 4d) nor their sizes (Figure 4e) was found to change in the G1 phase. Interestingly, despite the constant sizes of the CTCF foci, both the single-molecule detection counts (Appendix Figure S13a) and the molecule density (Appendix Figure S13b) in the CTCF foci were reduced from the mid-G1 phase to the G1/S transition, suggesting that CTCF dissociated from DNA during the transcription-dependent chromatin structural re-organization process. This STORM-based finding is consistent with a previous single-molecule study showing that binding of CTCF to chromatin decreases from the G1 phase to the S phase [61], as well as a Hi-C study showing that transcription elongation can disrupt CTCF-anchored chromatin loops [57].

In contrast to CTCF, MCM2 and PCNA showed drastically different patterns. In the G1 phase, MCM2 formed small clusters that distributed relatively around the TADs (Figure 4b, d). Intriguingly, at the G1/S transition, MCM2 foci became significantly larger (Figure 4b, e) and distributed more toward the center of the TADs (Figure 4b, d). Quantitative analyses of the foci showed that, while the single-molecule detection counts in the MCM2 foci increased from the 7 mid-G1 phase to the G1/S transition (Appendix Figure S13a), the molecule density decreased (Appendix Figure S13b). This observation suggested that MCM2 gradually became associated

with chromatin in the G1 phase, in line with the results by Gilbert and his colleagues [62]. As a replication elongation factor of the initiation complex, PCNA binds a subset of origins with 
411 the pre-IC complex and recruits DNA polymerases. In the G1 phase, we found that PCNA 412 formed small clusters around the TADs (Figure 4c, d). However, unlike MCM2, the PCNA foci 413 remained surrounding the TADs at the beginning of the S phase (Figure 4c, d). This spatial 414 distribution of PCNA provides a plausible reason why origins at the TAD periphery get 415 preferentially initiated. Moreover, from the mid-G1 phase to the G1/S transition, the size of the 416 PCNA foci was nearly doubled (Figure 4c, e) with both the single molecule detection counts 417 and the molecule density in the foci increased dramatically (Appendix Figure S13a \& b). These 418 data suggested that PCNA was gradually recruited to chromatin DNA, consistent with the 419 previous reports that PCNA clusters are much more visible by live cell imaging in the S phase 420 in comparison with the G1 phase [63-66].

\section{DISCUSSION}

Here, we unveiled a new mechanism for replication origin selection by directly visualizing 424 individual TADs and the spatial distribution and dynamics of replication origins in the TADs 425 using super-resolution imaging. We first found that replication initiation generally takes place 426 separately at the spatial boundary of the TAD (Figure 1 and Appendix Figure S5). Next, we 427 discovered that origins undergo relocalization along with the structural re-organization within 428 the TAD in the G1 phase, and the origins that either relocate to (e.g. ORI2 and ORI3) or remain 429 at (e.g. ORI1) the spatial boundary of the TAD are of higher replication efficiency (Figure 2 430 and Appendix Figure S9). Importantly, we found that chromatin structural re-organization 431 within the TADs is driven by disruption of chromatin loops during transcription elongation [57] 432 in the G1 phase (Figure 3 and Appendix Figure S10). Lastly, we observed that the major 433 replication machinery protein PCNA, which was previously found to be immobile in the S 434 phase [63-66], remains surrounding the TADs from the mid-G1 phase to the $S$ phase and 435 provides the origins exposed at the spatial boundary of a TAD with a better chance of accessing 436 the replication machinery.

\section{The "Chromatin Re-organization Induced Selective Initiation" (CRISI) Model}

439 Based on our results, we propose a "Chromatin Re-organization Induced Selective 440 Initiation" (CRISI) model (Figure 5) for replication origin selection. The CRISI model suggests 441 that the spatial localization of an origin in a TAD determines its replication efficiency. 442 Dynamically, in the early-to-mid G1 phase, all potential origins distribute homogeneously in 443 the TAD (Figure. 5a). Upon the onset of transcription, the chromatin loops in the TAD are de444 compacted and some loop anchors are disrupted, leading to a subset of origins relocalizing from 445 the inside of the TAD to the periphery (Figure 5b, c). Meanwhile, PCNA forms clusters that 446 remain around the TAD from the mid-G1 phase to the G1/S transition. The peripherally and 447 separately located origins are more accessible to the surrounding PCNA clusters and thus 
become active origins (Figure 5c). The distribution of active origins in TADs in our CRISI

449 model is in contrary with the classic Rosette model, which proposes that the active origins

450 cluster and co-fire in the chromatin domain [4].

451 Recently, based on the observation that nearly all replicons are spatially separated at the

452 beginning of the $\mathrm{S}$ phase, Cardoso and his colleagues also questioned the Rosette model [23].

453 They proposed a stochastic, proximity-induced replication initiation model, describing induced

454 domino-like origin activation that may lead to the temporal grouping of active replicons within

455 a chromatin fibre [28]. Nevertheless, as the replication origins were not imaged along with the

456 chromatin domains, how the origins are spatially organized in the chromatin domain and

457 whether the distribution can differentiate the origin efficiency were not known. In the current

458 work, we realized the first direct visualization and quantification of the relative localization and

459 organization of replication origins within individual TADs. Given that a TAD is typically $\sim 200$

$460 \mathrm{~nm}$ in radius (Figure 2d and Appendix Figure S9c) and the difference in barycenter distances

461 between active and dormant origins is less than $100 \mathrm{~nm}$ (Figure 2c and Appendix Figure S9b),

462 such quantification would require simultaneous imaging of both individual TADs and the

463 associated replication origins with nanometer spatial resolution. Therefore, 3D STORM with

$46420 \mathrm{~nm}$ lateral and $50 \mathrm{~nm}$ axial resolution would be more suitable for such analyses.

465 While aphidicolin is commonly used in the investigation of DNA replication [8-10], it is

466 concerned that such replication stress may stimulate the engagement of dormant origins in the

467 activated replication domains [6]. In our study, to avoid selection of abnormally activated

468 dormant origins, we combined the dynamic replication profile measured by BrdU-seq under

469 aphidicolin treatment and the replication landscape measured by OK-seq without aphidicolin

470 treatment. With this strategy, we were able to select dormant replication origins (ORI4 and

471 ORI5), which were not affected by aphidicolin treatment, for FISH labeling in the TADs. For

472 metabolic labeling of replication initiation sites with aphidicolin treatment, although some

473 dormant origins have the chance to be fired under the replication stress (Figure 1), it does not

474 affect the conclusion that replication starts at the periphery of the chromatin domains.

475 Regarding the constrained mobility of PCNA clusters in the nucleus, we speculate three

476 possible mechanisms that are not mutually exclusive. Firstly, PCNA and the replisomes are

477 giant complexes binding DNA with low diffusive mobility. Secondly, PCNA and the replisomes

478 may be attached to the nuclear matrix (Figure 5c), which is supported by an immunoelectron

479 microscopy study showing that DNA polymerase a, PCNA, and nascent DNA are colocalized

480 in nucleoskeleton bodies [67]. Thirdly, the proteins comprising replisome complexes might

481 form liquid condensates that phase-separate from TADs [68]. These possibilities would be

482 interesting subjects for future studies.

483 That the chromatin structural dynamics within the TADs make origins accessible to 484 immobile PCNA clusters provides an interesting viewpoint to understand protein-DNA 
interactions in the nucleus, which are commonly considered to be based on diffusive search of proteins such as transcription factors on chromatin DNA $[69,70]$. Such mechanisms may be involved in various nuclear functions. For example, during DNA damage repair, ATM is restricted at the double strand breaking (DSB) site while phosphorylation of H2AX by ATM spreads over a domain [71]. The discrepancy between the distribution of the kinase (ATM) and its product $(\gamma \mathrm{H} 2 \mathrm{AX})$ can be explained by the local movements of the chromatin fiber inside the TAD which bring distant nucleosomes to spatial proximity of ATM [71]. In the future, other imaging methods such as sequential imaging approach (Hi-M) may be combined with oligoSTORM to further investigate chromosome organization and functions in single nuclei [72].

\section{Replication, transcription, and chromatin structure}

It has been known for many years that transcription is profoundly related to replication $[73,74]$. However, while transcription is known to be highly correlated with the replication timing of TADs $[58,75]$, it is unclear whether transcription regulates origin selection within individual TADs. Intriguingly, although the genetic and epigenetic signatures of active origins mapped by various methods seem quite different and hierarchical [2], they are mostly markers of active transcription and interdependent in the context of transcription. Transcription has been reported to change chromatin structure at different levels. Our imaging data reveal that the transcription activities can displace CTCF from the TADs (Appendix Figure S13) and decompact the chromatin domain (Figure 2d\&3e), consistent with the previously reported Hi-C data $[49,57]$. These effects, together with other transcription-induced changes in nucleosomes, chromatin fibers and enhancer-promoter loops, re-organize the chromatin structures within the TADs to relocate a subset of origins to the TAD periphery and consequently, these origins possess higher replication efficiency for being more accessible to the peri-TAD PCNA clusters. The transcription-dependent CRISI model predicts that enhancing transcription activity should increase the selectivity of replication origins, while repressing transcription should cause the opposite effect. Indeed, two recent studies of replication initiation in particular genes showed that enhanced transcription leads to more selective initiation of origins $[54,76]$ while transcription inhibition causes more origins to be used for replication [77]. However, while our results explain why a subset of origins is preferentially activated in a TAD, the envision that how specific origins are relocated to the TAD periphery by transcription activity is unclear.

7 Further investigations using CRISPRi [78] to interfere with the transcription of specific genes and observation of changes in the replication efficiency of origins associated with these genes 9 would illuminate the detailed mechanisms underlying the non-random, yet flexible, nature of replication origin selection. 
522 source of genome instability $[73,79]$. Therefore, how cells prevent or resolve the transcript-

523 replication conflicts has been an important question. One major mechanism is to temporally

524 separate transcription and replication for the same genomic regions [80]. Our data suggested

525 that the replication machineries are confined around the TAD and spatially separated from the

526 transcription machineries, which mainly function within the TAD. Therefore, our work provides

527 a new mechanism for cells to avoid the conflicts between replication and transcription based

528 on spatial/topological separation.

529 In summary, the CRISI model demonstrates important coordination among DNA

530 replication, transcription, and chromatin structure, which reconciles the discrepancy of different

531 signatures for origin efficiency. Lastly, our work also provides new insights into how 3D

532 genome structural dynamics, particularly the intra-TAD physical structures, may regulate other

533 nuclear processes on chromatin templates such as DNA repair, adding a new layer of

534 understanding to chromatin structure and functions.

535

536

537 Supplementary Information is available in the online version of the paper.

538

539

540

Acknowledgements The authors thank Dr. Wei Guo (University of Pennsylvania) for the HeLa-S3 cell line, Florian Levet (University of Bordeaux) for SR-Tesseler analysis and Ruifeng $\mathrm{Li}$ (Peking University) for the Hi-C interaction map. This work was supported by grants from the National Key R\&D Program of China (No. 2017YFA0505300), the National Natural Science Foundation of China (21573013), and the National Science Fund for Distinguished Young Scholars (21825401 to Y.S.). Grants for G.L. from the Ministry of Science and Technology of China (2017YFA0504200) and the Chinese Academy of Sciences (CAS) Strategic Priority Research Program (XDB19040202).

Author Contributions Y.L., B.X. and Y.S. conceived the project and designed the experiments. Y.L. performed the probe synthesis, sample preparation, imaging and data analysis. B.X. performed coding for the data analysis algorithms. H.L. performed the sequencing experiments and L.Z. performed the sequencing data analysis. Y.L. and Y.S. wrote the manuscript. The other authors took part in experiments and data analysis with Y.L.

Author Information Reprints and permissions information is available at. The authors declare no competing financial interests. Readers are welcome to comment on the online version of the paper. Correspondence and requests for materials should be addressed to Y.S. (sun_yujie@pku.edu.cn). 
559

560

561

562

563

564

565

566

567

568

569

570

571

572

\section{References}

1. Macheret M, Halazonetis TD: DNA replication stress as a hallmark of cancer. Annu Rev Pathol 2015, 10:425-448

2. Mechali M: Eukaryotic DNA replication origins: many choices for appropriate answers. Nat Rev Mol Cell Biol 2010, 11:728-738.

3. Hyrien O: Peaks cloaked in the mist: the landscape of mammalian replication origins. I Cell Biol 2015, 208:147-160.

4. Fragkos $\mathrm{M}$, Ganier $\mathrm{O}$, Coulombe $\mathrm{P}$, Mechali M: DNA replication origin activation in space and time. Nat Rev Mol Cell Biol 2015, 16:360-374.

5. Rivera-Mulia JC, Gilbert DM: Replicating Large Genomes: Divide and Conquer. Mol Cell 2016, 62:756-765.

6. Ge XQ, Jackson DA, Blow JJ: Dormant origins licensed by excess $M c m$ 2-7 are required for human cells to survive replicative stress. Genes Dev 2007, 21:3331-3341.

7. Ibarra A, Schwob E, Mendez J: Excess MCM proteins protect human cells from replicative stress by licensing backup origins of replication. Proc Natl Acad Sci U S A 2008, 105:8956-8961.

8. Huberman JA, Riggs AD: On the Mechanism of DNA Replication in Mammalian Chromosomes. $J$ Mol Biol 1968, 32:327-341.

9. Jackson DA, Pombo A: Replicon clusters are stable units of chromosome structure: evidence that nuclear organization contributes to the efficient activation and propagation of $S$ phase in human cells. J Cell Biol 1998, 140:1285-1295.

10. Bensimon RLaA: DNA Replication Origin Interference Increases the Spacing Between Initiation Events in Human Cells. Mol Biol Cell 2006.

11. Chen C, Xing D, Tan L, Li H, Zhou G, Huang L, Xie XS: Single-cell whole-genome analyses by Linear Amplification via Transposon Insertion (LIANTI). Science 2017, 356:189.

12. Dileep V, Gilbert DM: Single-cell replication profiling to measure stochastic variation in mammalian replication timing. Nature Communications 2018, 9:427.

13. Marks AB, Smith OK, Aladjem MI: Replication origins: determinants or consequences of nuclear organization? Curr Opin Genet Dev 2016, 37:67-75.

14. Smith OK, Aladjem MI: Chromatin structure and replication origins: determinants of chromosome replication and nuclear organization. $J$ Mol Biol 2014, 426:3330-3341.

15. Vogelstein B, Pardoll DM, Coffey DS: Supercoiled Loops and Eucaryotic DNA Replication. Cell 1980, 80:90156-90157.

16. Buongiorno-Nardelli M, Micheli G, Carri MT, Marilley M: A relationship between replicon size and supercoiled loop domains in the eukaryotic genome. Nature 1982, 298:100-102.

17. Courbet S, Gay S, Arnoult N, Wronka G, Anglana M, Brison O, Debatisse M: Replication fork movement sets chromatin loop size and origin choice in mammalian cells. Nature 2008, 455:557560.

18. Pope BD, Ryba T, Dileep V, Yue F, Wu W, Denas O, Vera DL, Wang Y, Hansen RS, Canfield TK, et al: Topologically associating domains are stable units of replication-timing regulation. Nature 2014, 515:402-405.

19. Dixon JR, Gorkin DU, Ren B: Chromatin Domains: The Unit of Chromosome Organization. Mol Cell 2016, 62:668-680.

20. Nakayasu H, Berezney R: Mapping replicational sites in the eucaryotic cell nucleus. J Cell Biol 1989, 108:1-11.

21. Ma H, Samarabandu J, Devdhar RS, Acharya R, Cheng P-c, Meng C, Berezney R: Spatial and Temporal Dynamics of DNA Replication Sites in Mammalian Cells. The Journal of Cell Biology 1998, 143:14151425.

22. Visser AE, Eils R, Jauch A, Little G, Bakker PJ, Cremer T, Aten JA: Spatial distributions of early and late replicating chromatin in interphase chromosome territories. Exp Cell Res 1998, 243:398-407.

23. Chagin VO, Casas-Delucchi CS, Reinhart M, Schermelleh L, Markaki Y, Maiser A, Bolius JJ, Bensimon A, Fillies $M$, Domaing $P$, et al: $4 D$ Visualization of replication foci in mammalian cells corresponding to individual replicons. Nat Commun 2016, 7:11231.

24. Su QP, Zhao ZW, Meng L, Ding M, Zhang W, Li Y, Liu M, Li R, Gao Y-Q, Xie XS, Sun Y: CTCF-mediated Chromatin Structures Dictate the Spatio-temporal Propagation of Replication Foci. bioRxiv 2019:525915.

25. Xiang W, Roberti MJ, Heriche JK, Huet S, Alexander S, Ellenberg J: Correlative live and super- 
resolution imaging reveals the dynamic structure of replication domains. J Cell Biol 2018, 217:1973-1984.

26. Cayrou C, Coulombe P, Vigneron A, Stanojcic S, Ganier O, Peiffer I, Rivals E, Puy A, Laurent-Chabalier $S$, Desprat $R$, Mechali M: Genome-scale analysis of metazoan replication origins reveals their organization in specific but flexible sites defined by conserved features. Genome Res 2011, 21:1438-1449.

27. Guillou E, Ibarra A, Coulon V, Casado-Vela J, Rico D, Casal I, Schwob E, Losada A, Mendez J: Cohesin organizes chromatin loops at DNA replication factories. Genes Dev 2010, 24:2812-2822.

28. Lob D, Lengert N, Chagin VO, Reinhart M, Casas-Delucchi CS, Cardoso MC, Drossel B: 3D replicon distributions arise from stochastic initiation and domino-like DNA replication progression. Nat Commun 2016, 7:11207.

29. Boettiger AN, Bintu B, Moffitt JR, Wang S, Beliveau BJ, Fudenberg G, Imakaev M, Mirny LA, Wu CT, Zhuang $X$ : Super-resolution imaging reveals distinct chromatin folding for different epigenetic states. Nature 2016, 529:418-422.

30. Beliveau BJ, Boettiger AN, Nir G, Bintu B, Yin P, Zhuang X, Wu CT: In Situ Super-Resolution Imaging of Genomic DNA with OligoSTORM and OligoDNA-PAINT. Methods Mol Biol 2017, 1663:231-252.

31. Nir G, Farabella I, Perez Estrada C, Ebeling CG, Beliveau BJ, Sasaki HM, Lee SD, Nguyen SC, McCole $\mathrm{RB}$, Chattoraj $\mathrm{S}$, et al: Walking along chromosomes with super-resolution imaging, contact maps, and integrative modeling. PLoS Genet 2018, 14:e1007872.

32. Beliveau BJ, Joyce EF, Apostolopoulos N, Yilmaz F, Fonseka CY, McCole RB, Chang Y, Li JB, Senaratne $\mathrm{TN}$, Williams $\mathrm{BR}$, et al: Versatile design and synthesis platform for visualizing genomes with Oligopaint FISH probes. Proc Natl Acad Sci U S A 2012, 109:21301-21306.

33. Rust MJ, Bates $M$, Zhuang $X$ : Sub-diffraction-limit imaging by stochastic optical reconstruction microscopy (STORM). Nature Methods 2006, 3:793-795.

34. Betzig E, Patterson GH, Sougrat R, Lindwasser OW, Olenych S, Bonifacino JS, Davidson MW, Lippincott-Schwartz J, Hess HF: Imaging intracellular fluorescent proteins at nanometer resolution. Science 2006, 313:1642-1645.

35. Hess ST, Girirajan TP, Mason MD: Ultra-high resolution imaging by fluorescence photoactivation localization microscopy. Biophys J 2006, 91:4258-4272.

36. Schermelleh L, Ferrand A, Huser T, Eggeling C, Sauer M, Biehlmaier O, Drummen GPC: Superresolution microscopy demystified. Nat Cell Biol 2019, 21:72-84.

37. Mateo LJ, Murphy SE, Hafner A, Cinquini IS, Walker CA, Boettiger AN: Visualizing DNA folding and RNA in embryos at single-cell resolution. Nature 2019, 568:49-54.

38. Bintu B, Mateo LJ, Su JH, Sinnott-Armstrong NA, Parker M, Kinrot S, Yamaya K, Boettiger AN, Zhuang $X$ : Super-resolution chromatin tracing reveals domains and cooperative interactions in single cells. Science 2018, 362.

39. Dimitrova DS, Todorov IT, Melendy T, Gilbert DM: Mcm2, but not RPA, is a component of the mammalian early G1-phase prereplication complex. The Journal of cell biology 1999, 146:709-722.

40. Su QP, Zhao ZW, Meng L, Ding M, Zhang W, Li Y, Liu M, Li R, Gao Y-Q, Xie XS, Sun Y: 2019.

41. Levet F, Hosy E, Kechkar A, Butler C, Beghin A, Choquet D, Sibarita JB: SR-Tesseler: a method to segment and quantify localization-based super-resolution microscopy data. Nat Methods 2015, 12:1065-1071.

42. Ester M, Kriegel H-P, Sander J, Xu X: A density-based algorithm for discovering clusters in large spatial databases with noise. In KDD-96 Proceedings. 1996: 226-231.

43. E. M. M. MANDERS JS, G. J. BRAKENHOFF, R. VAN DRIEL and J. A. ATEN: Dynamics of threedimensional replication patterns during the S-phase, analysed by double labelling of DNA and confocal microscopy. Journal of Cell Science 1992.

44. Takebayashi SI, Ogata M, Okumura K: Anatomy of Mammalian Replication Domains. Genes (Basel) $2017,8$.

45. Boos D, Ferreira P: Origin Firing Regulations to Control Genome Replication Timing. Genes (Basel) 2019, 10.

46. Farkash-Amar S, Lipson D, Polten A, Goren A, Helmstetter C, Yakhini Z, Simon I: Global organization of replication time zones of the mouse genome. Genome Res 2008, 18:1562-1570.

47. Long H, Zhang L, Lv M, Wen Z, Zhang W, Chen X, Zhang P, Li T, Chang L, Jin C, et al: H2A.Z facilitates licensing and activation of early replication origins. Nature 2019.

48. Petryk N, Kahli M, d'Aubenton-Carafa Y, Jaszczyszyn Y, Shen Y, Silvain M, Thermes C, Chen CL, Hyrien O: Replication landscape of the human genome. Nat Commun 2016, 7:10208. 
49. Nagano T, Lubling Y, Varnai C, Dudley C, Leung W, Baran Y, Mendelson Cohen N, Wingett S, Fraser P, Tanay A: Cell-cycle dynamics of chromosomal organization at single-cell resolution. Nature 2017, 547:61-67.

50. Gilbert VDaDM: Topologically-associating domains and their long-range contacts are established during early $\mathrm{G} 1$ coincident with the establishment of the replication timing program. Genome Res 2015.

51. Ong C-T, Corces VG: CTCF: an architectural protein bridging genome topology and function. Nature reviews Genetics 2014, 15:234-246.

52. Rao SSP, Huang SC, Glenn St Hilaire B, Engreitz JM, Perez EM, Kieffer-Kwon KR, Sanborn AL, Johnstone SE, Bascom GD, Bochkov ID, et al: Cohesin Loss Eliminates All Loop Domains. Cell 2017, 171:305320 e324.

53. Nora EP, Goloborodko A, Valton AL, Gibcus JH, Uebersohn A, Abdennur N, Dekker J, Mirny LA, Bruneau BG: Targeted Degradation of CTCF Decouples Local Insulation of Chromosome Domains from Genomic Compartmentalization. Cell 2017, 169:930-944 e922.

54. Chen YH, Keegan S, Kahli M, Tonzi P, Fenyo D, Huang TT, Smith DJ: Transcription shapes DNA replication initiation and termination in human cells. Nat Struct Mol Biol 2019, 26:67-77.

55. Rando OJ, Chang HY: Genome-wide views of chromatin structure. Annual review of biochemistry 2009, 78:245-271.

56. Kornberg RD, Lorch Y: Chromatin Structure and Transcription. Annual Review of Cell Biology 1992, 8:563-587.

57. Heinz S, Texari L, Hayes MGB, Urbanowski M, Chang MW, Givarkes N, Rialdi A, White KM, Albrecht RA, Pache L, et al: Transcription Elongation Can Affect Genome 3D Structure. Cell 2018, 174:15221536 e1522.

58. Sima J, Chakraborty A, Dileep V, Michalski M, Klein KN, Holcomb NP, Turner JL, Paulsen MT, RiveraMulia JC, Trevilla-Garcia C, et al: Identifying cis Elements for Spatiotemporal Control of Mammalian DNA Replication. Cell 2019, 176:816-830 e818.

59. Yankulov K, Yamashita K, Roy R, Egly JM, Bentley DL: The transcriptional elongation inhibitor 5,6dichloro-1-beta-D-ribofuranosylbenzimidazole inhibits transcription factor IIH-associated protein kinase. J Biol Chem 1995, 270:23922-23925.

60. Mailand N, Gibbs-Seymour I, Bekker-Jensen S: Regulation of PCNA-protein interactions for genome stability. Nature Reviews Molecular Cell Biology 2013, 14:269.

61. Agarwal H, Reisser M, Wortmann C, Gebhardt JCM: Direct Observation of Cell-Cycle-Dependent Interactions between CTCF and Chromatin. Biophys J 2017, 112:2051-2055.

62. Gilbert DSDDM: Mcm2, but Not RPA, Is a Component of the Mammalian Early G1-Phase Prereplication Complex. The Journal of Cell Biology, 1999.

63. Essers J, Theil AF, Baldeyron C, van Cappellen WA, Houtsmuller AB, Kanaar R, Vermeulen W: Nuclear dynamics of PCNA in DNA replication and repair. Molecular and cellular biology 2005, 25:93509359.

64. Leonhardt H, Rahn HP, Weinzierl P, Sporbert A, Cremer T, Zink D, Cardoso MC: Dynamics of DNA replication factories in living cells. The Journal of cell biology 2000, 149:271-280.

65. Sporbert A, Gahl A, Ankerhold R, Leonhardt H, Cardoso MC: DNA Polymerase Clamp Shows Little Turnover at Established Replication Sites but Sequential De Novo Assembly at Adjacent Origin Clusters. Molecular Cell 2002, 10:1355-1365.

66. Zessin PJM, Sporbert A, Heilemann M: PCNA appears in two populations of slow and fast diffusion with a constant ratio throughout S-phase in replicating mammalian cells. Scientific Reports 2016, 6:18779.

67. Hozák P, Hassan AB, Jackson DA, Cook PR: Visualization of replication factories attached to a nucleoskeleton. Cell 1993, 73:361-373.

68. Shin Y, Chang Y-C, Lee DSW, Berry J, Sanders DW, Ronceray P, Wingreen NS, Haataja M, Brangwynne CP: Liquid Nuclear Condensates Mechanically Sense and Restructure the Genome. Cell 2018, 176:1518.

69. Gebhardt JCM, Suter DM, Roy R, Zhao ZW, Chapman AR, Basu S, Maniatis T, Xie XS: Single-molecule imaging of transcription factor binding to DNA in live mammalian cells. Nature Methods 2013, 10:421-426.

70. Liu Z, Tjian R: Visualizing transcription factor dynamics in living cells. The Journal of cell biology 2018, 217:1181-1191.

71. Caron P, Aymard F, lacovoni JS, Briois S, Canitrot Y, Bugler B, Massip L, Losada A, Legube G: Cohesin 

8

Protects Genes against pH2AX Induced by DNA Double-Strand Breaks. PLOS Genetics 2012, 8:e1002460.

72. Cardozo Gizzi AM, Cattoni DI, Fiche JB, Espinola SM, Gurgo J, Messina O, Houbron C, Ogiyama Y, Papadopoulos GL, Cavalli G, et al: Microscopy-Based Chromosome Conformation Capture Enables Simultaneous Visualization of Genome Organization and Transcription in Intact Organisms. Mol Cell 2019, 74:212-222 e215.

73. Garcia-Muse T, Aguilera A: Transcription-replication conflicts: how they occur and how they are resolved. Nat Rev Mol Cell Biol 2016, 17:553-563.

74. Achar YJ, Foiani M: Coordinating Replication with Transcription. Adv Exp Med Biol 2017, 1042:455487.

75. Rivera-Mulia JC, Gilbert DM: Replication timing and transcriptional control: beyond cause and effect-part III. Curr Opin Cell Biol 2016, 40:168-178.

76. Blin M, Le Tallec B, Nahse V, Schmidt M, Brossas C, Millot GA, Prioleau MN, Debatisse M: Transcription-dependent regulation of replication dynamics modulates genome stability. Nat Struct Mol Biol 2019, 26:58-66.

77. Macheret M, Halazonetis TD: Intragenic origins due to short $\mathbf{G 1}$ phases underlie oncogene-induced DNA replication stress. Nature 2018, 555:112.

78. Qi LS, Larson MH, Gilbert LA, Doudna JA, Weissman JS, Arkin AP, Lim WA: Repurposing CRISPR as an RNA-guided platform for sequence-specific control of gene expression. Cell 2013, 152:1173-1183.

79. Hamperl S, Bocek MJ, Saldivar JC, Swigut T, Cimprich KA: Transcription-Replication Conflict Orientation Modulates R-Loop Levels and Activates Distinct DNA Damage Responses. Cell 2017, 170:774-786.e719.

80. Gilbert DM: Replication timing and transcriptional control: beyond cause and effect. Curr Opin Cell Biol 2002, 14:377-383.

81. Petryk N, Kahli M, d'Aubenton-Carafa Y, Jaszczyszyn Y, Shen Y, Silvain M, Thermes C, Chen C-L, Hyrien O: Replication landscape of the human genome. Nature Communications 2016, 7:10208.

82. Jackson DA, Pombo A: Replicon Clusters Are Stable Units of Chromosome Structure: Evidence That Nuclear Organization Contributes to the Efficient Activation and Propagation of $S$ Phase in Human Cells. The Journal of Cell Biology 1998, 140:1285.

83. Dileep V, Ay F, Sima J, Vera DL, Noble WS, Gilbert DM: Topologically associating domains and their long-range contacts are established during early G1 coincident with the establishment of the replication-timing program. Genome research 2015, 25:1104-1113.

84. Wu P, Li T, Li R, Jia L, Zhu P, Liu Y, Chen Q, Tang D, Yu Y, Li C: 3D genome of multiple myeloma reveals spatial genome disorganization associated with copy number variations. Nature Communications 2017, 8:1937.

85. Guillou E, Ibarra A, Coulon V, Casado-Vela J, Rico D, Casal I, Schwob E, Losada A, Méndez J: Cohesin organizes chromatin loops at DNA replication factories. Genes \& Development 2010, 24:2812-2822.

86. Rust MJ, Bates $M$, Zhuang $X$ : Sub-diffraction-limit imaging by stochastic optical reconstruction microscopy (STORM). Nat Methods 2006, 3:793-795. 


\section{Figures and Figure Legends}

a

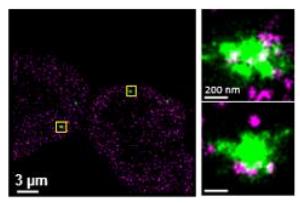

EdU 10 min TAD1

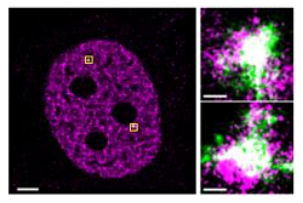

EdU 60 min TAD1

b

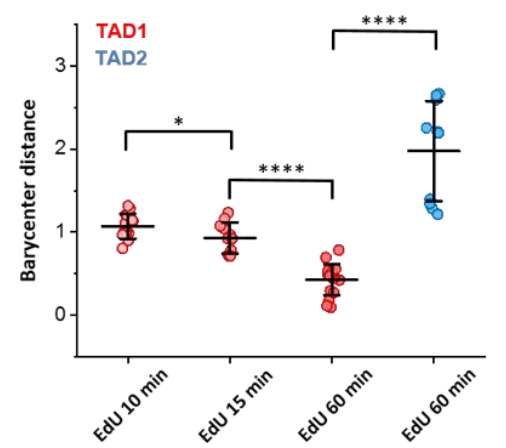

C

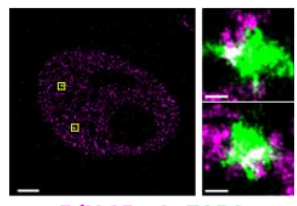

EdU 15 min TAD1

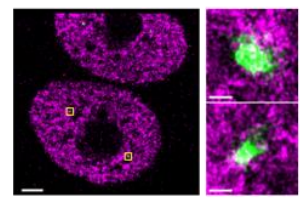

EdU 60 min TAD2

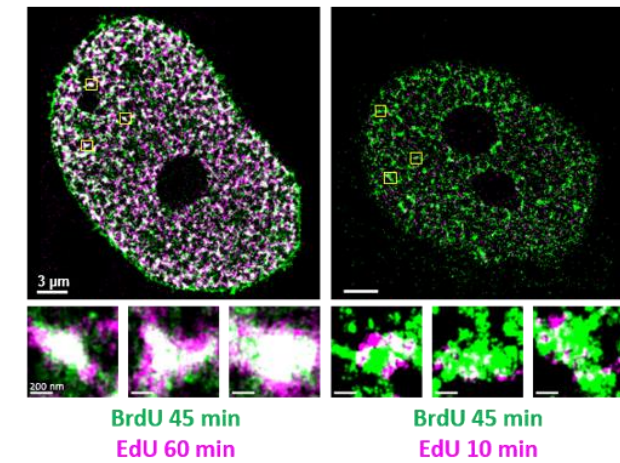

d

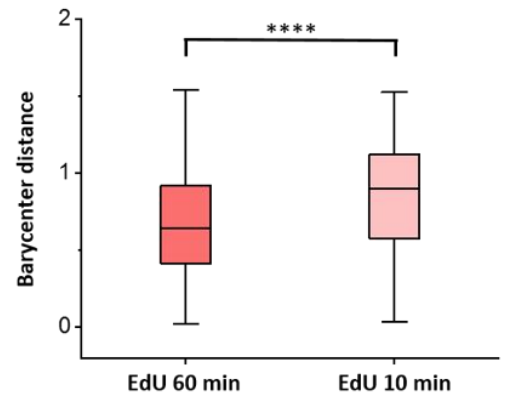

Figure 1. Super-resolution imaging of RFi and TADs in the S phase. a, Representative STORM images of TAD1 and TAD2 labeled by Oligopaint probes (green) and RFi labeled metabolically for different durations (purple) (Methods). TAD1 and TAD2 were chosen based on the replication timing profile and Hi-C interaction heatmap of HeLa cells (Appendix Figure S1). TAD1: an early replicating domain (Chr1:16911932-17714928). TAD2: a middle replicating domain (Chr1:17722716-18846245). Metabolic labeling of DNA replication was performed by supplying EdU to the cell upon release into the $\mathrm{S}$ phase for $10 \mathrm{~min}, 15 \mathrm{~min}$, and $60 \mathrm{~min}$ (purple). The areas inside the yellow squares are shown at higher magnification to the right of each nucleus. Portions of the two signals that overlap are shown in white. $\mathbf{b}$, Barycenter distances between the TAD and its spatially associated RFi (Methods) in a. Horizontal lines and error bars represent the mean values \pm s.d. of three or more independent biological replicates $(n=16$ cells). c, Representative STORM images of RFi labeled metabolically for different durations in two consecutive cell cycles. Consecutive metabolic labeling of DNA replication was performed by supplying BrdU (green) to the cell upon release into the $\mathrm{S}$ phase in the first cell cycle, followed by supplying EdU (purple) to the cell upon release into the $\mathrm{S}$ phase in the second cell cycle (for different durations). The areas inside the yellow squares are shown at higher magnification below each nucleus. d, Box plot of barycenter distances between BrdU and EdU labeled RFi in $\mathbf{c}$ (data were pooled from $\mathrm{n}=10$ cells). Center line, median; box limits, $25 \%$ and $75 \%$ of the entire population; whiskers, observations within $1.5 \times$ the interquartile range of the box limits. Significance was analyzed by un-paired two sample parametric $\mathrm{t}$ test. $* * * * \mathrm{P}<0.0001, * * * \mathrm{P}<0.0005, * * \mathrm{P}<0.01, * \mathrm{P}<0.05$, N.S.: not significant. 3D results are shown in Fig. S2\&S5. 

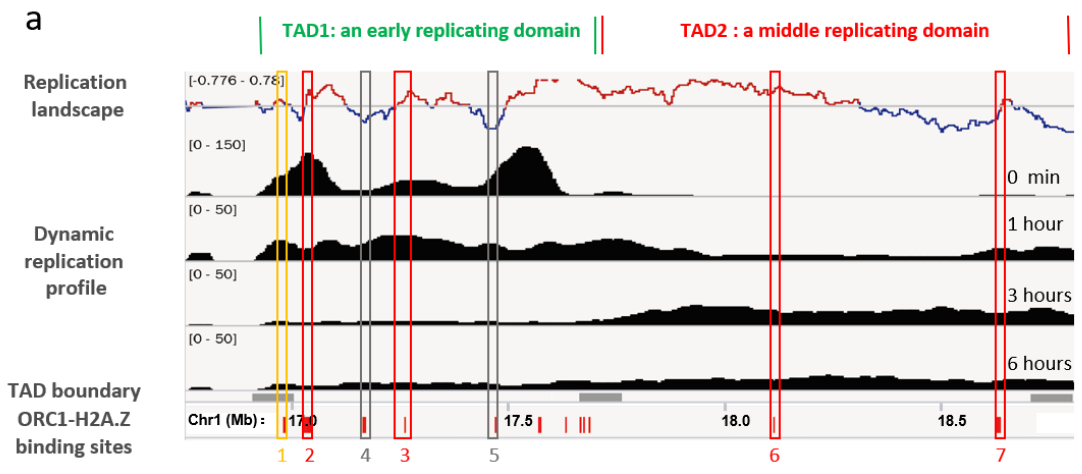

b
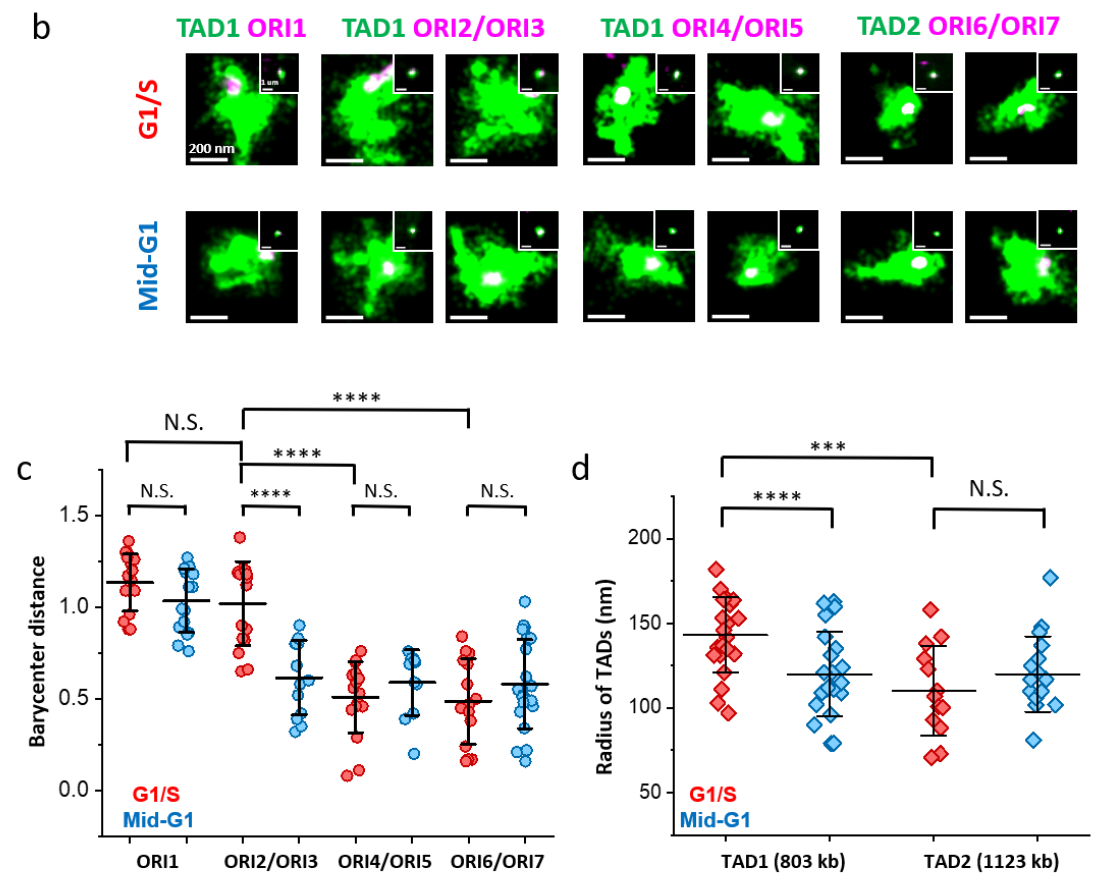

Figure 2. Spatial distribution of replication origins relative to the TADs in the G1 and G1/S phases. a, A scheme of replication in TAD1 and TAD2. The top profile represents the replication landscape obtained by OK-seq. (-0.776-0.78) was the threshold of OK-seq [81]. The middle black peaks represent the dynamic replication profile, which was obtained by 10-min BrdU pulse labeling at $0 \mathrm{~min}, 30 \mathrm{~min}, 3$ hours, and 6 hours into the S phase. $(0-50)$ or $(0-150)$ is the range of normalized BrdU-seq data. The grey bars represent the TAD boundaries in the RDs. The small red bars at the bottom represent the ORC1 and $\mathrm{H} 2 \mathrm{~A} . \mathrm{Z}$ binding sites indicating the potential replication origins. Representative active and dormant replication origins defined by the BrdU-seq data and the OK-seq profile are marked with vertical rectangles. Yellow rectangle: active replication origin (ORI1) at the TAD boundary. Red rectangles: active replication origins in TAD1 (ORI2 and ORI3) and TAD2 (ORI6 and ORI7). Black rectangles: dormant replication origins in TAD1 (ORI4 and ORI5). b, Representative STORM images of TADs (green) and their origins (purple) labeled by FISH with oligoprobes in the G1 and G1/S phases. Upper, TADs and origins labeled at the G1/S transition. Lower, TADs and origins labeled at approximately 5 hours into the G1 phase. Portions of the two signals that overlap are shown in white. The corresponding conventional images are shown in the inset. c, Barycenter distances between origins and TADs in $\mathbf{b}$ ( $\mathrm{n} \geq 10$ cells). $\mathbf{d}$, Radii of TAD1 and TAD 2 in the $\mathrm{G} 1$ or $\mathrm{G} 1 / \mathrm{S}$ phase $(\mathrm{n} \geq 10$ cells). For lines and statistics in $\mathbf{c}$ and $\mathbf{d}$ see the description in the legend of Figure 1. 3D results are shown in Fig. S9. 
a

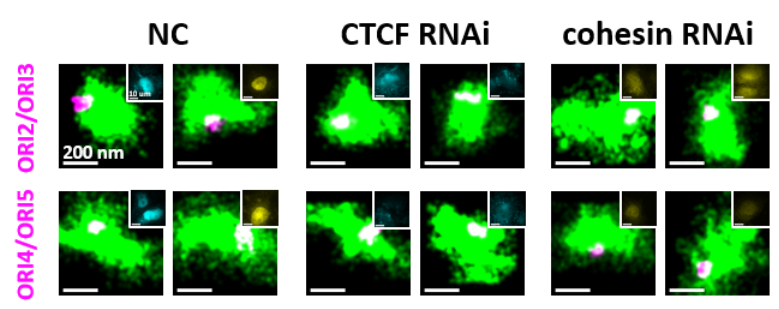

b

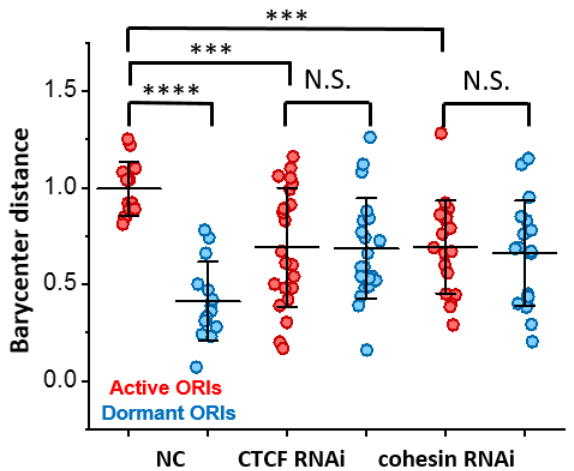

C

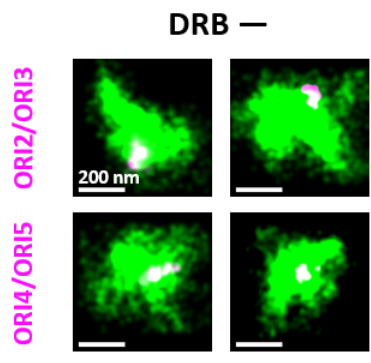

d

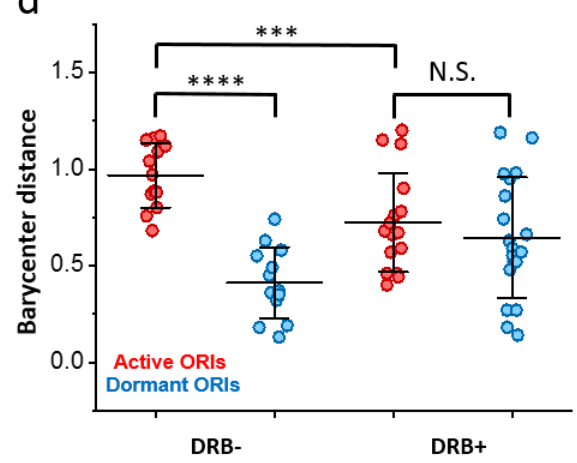

e

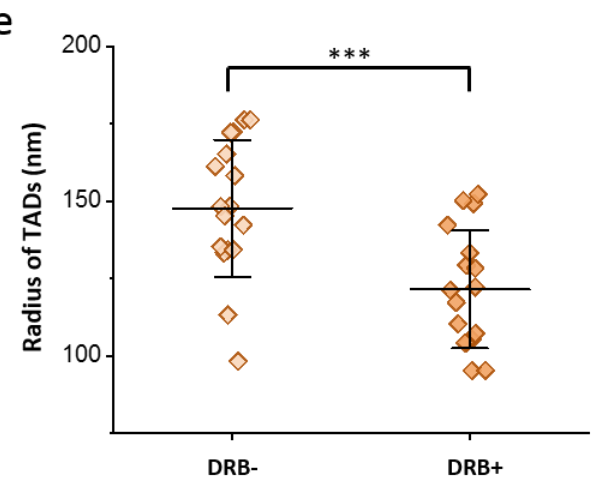

Figure 3. The spatial distribution of replication origins within the TADs at the G1/S transition is dependent on CTCF, cohesin and transcription. a, Representative STORM images of origins (purple) in TAD1 (green) after treatment of cells with the indicated siRNAs. Conventional images indicate the concentration of CTCF (cyan) or cohesin (yellow) in the nucleus. b, Barycenter distances between active or dormant origins in TAD1 after treatment of cells with the indicated siRNAs. Portions of the two signals that overlap are shown in white. c, Representative STORM images of origins (purple) in TAD1 (green). Left: no DRB. Right: with DRB. d, Barycenter distances between active/dormant origins and TAD1 with or without DRB treatment. e, Radius of TAD1 treated with or without DRB. For lines and statistics in $\mathbf{b}, \mathbf{d}$, and $\mathbf{e}$ see the description in the legend of Figure 1 ( $n \geq 10$ cells). 3D results are shown in Fig. S10. 


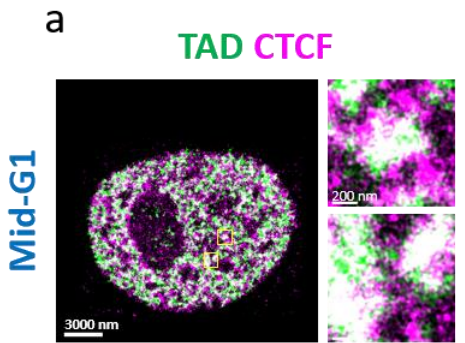

b
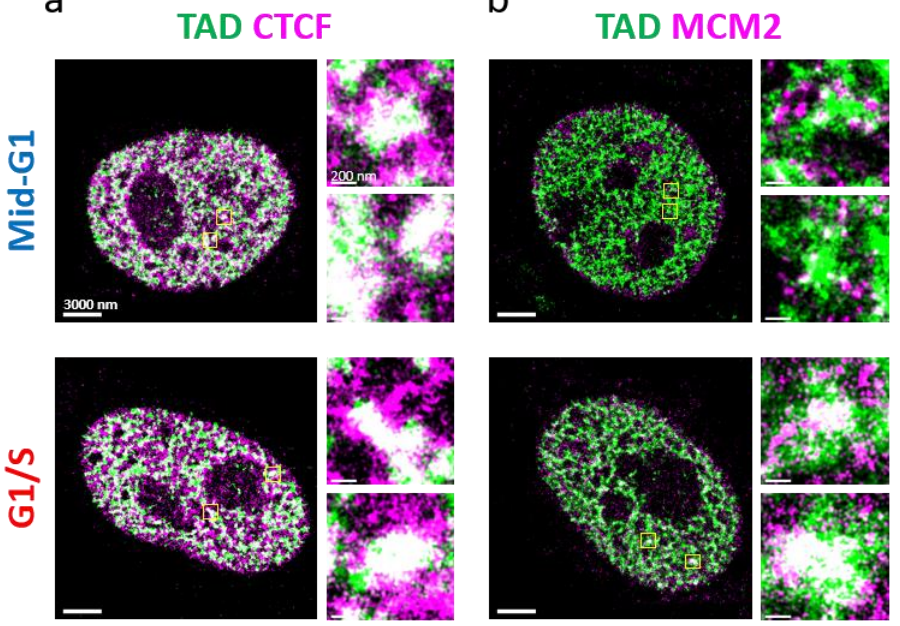

$\mathrm{C}$
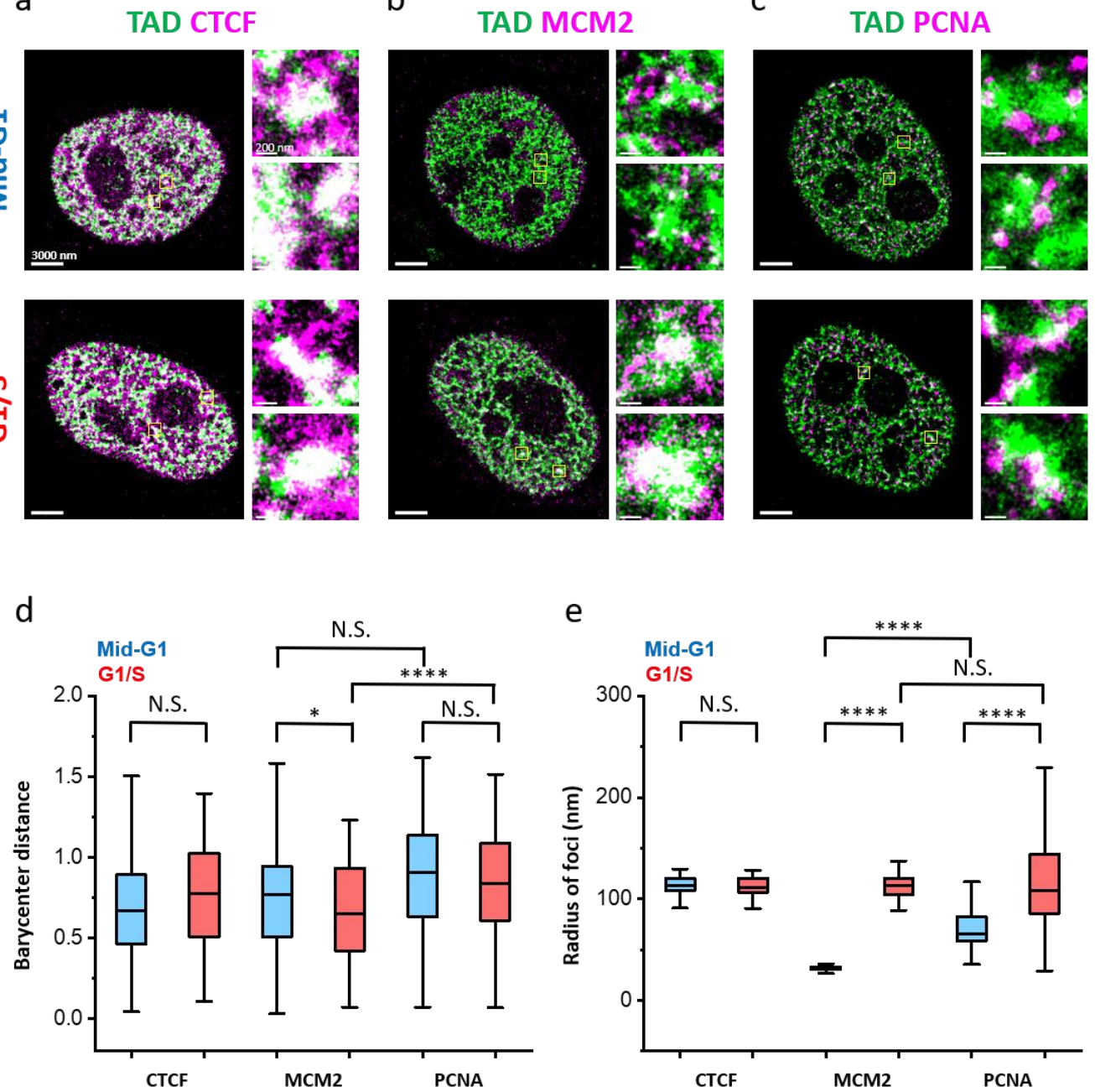

Figure 4. Spatial distributions of CTCF, MCM2 and PCNA relative to the early replicating TADs in the G1 and G1/S phases. a-c, Representative STORM images of CTCF, MCM2 and PCNA labeled by immunostaining (purple) and metabolically labeled TADs (green). Cells were fixed and labeled in the mid-G1 phase (upper) or G1/S phase (lower). The areas inside the yellow squares are shown at higher magnification next to each nucleus. Portions of the two signals that overlap are shown in white. d, Barycenter distances between CTCF, MCM2 or PCNA with the TADs in the mid-G1 phase or G1/S phase. e, Radii of CTCF, MCM2 or PCNA foci in the mid-G1 phase or $\mathrm{G} 1 / \mathrm{S}$ phase. For lines and statistics in $\mathbf{d}$ and $\mathbf{e}$ see the description in the legend of Figure $1(\mathrm{n}=10$ cells). 

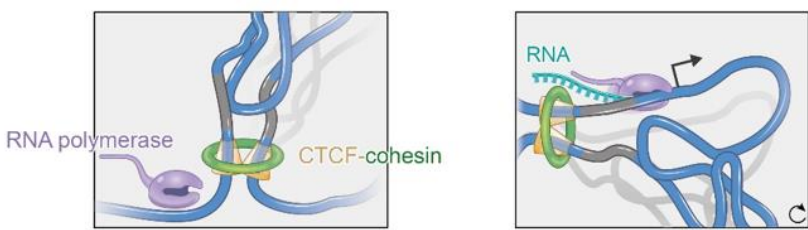

b

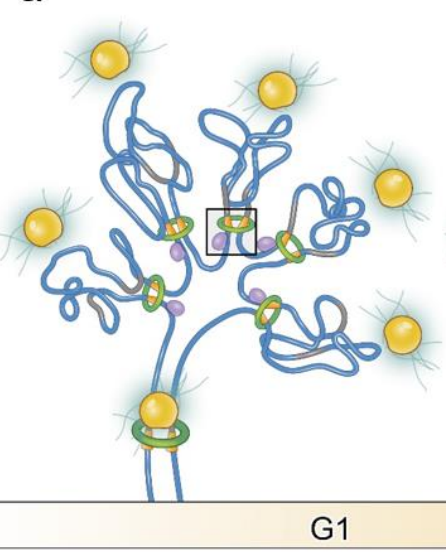

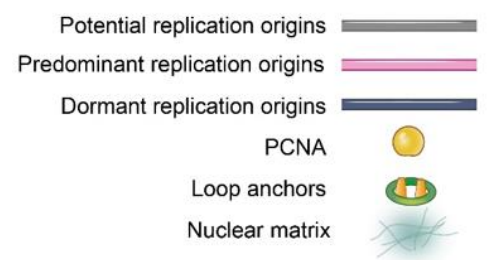

C

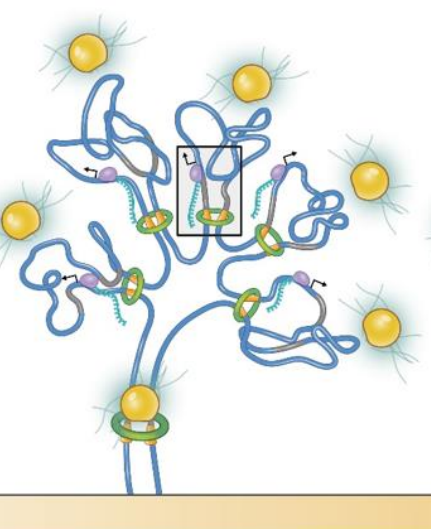

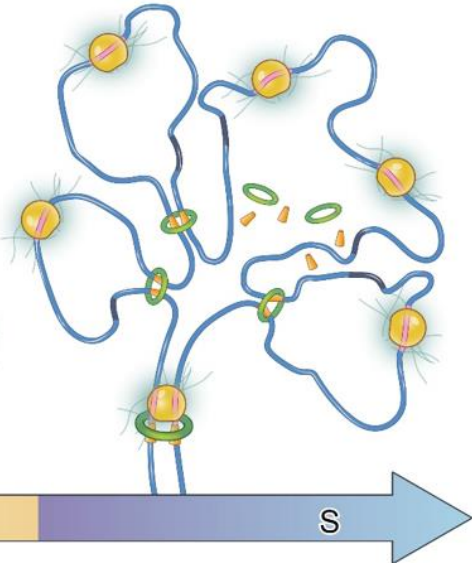

Figure 5. "Chromatin Re-organization Induced Selective Initiation" (CRISI) model for selective initiation of DNA replication origins. a, In the early G1 phase, the spatial distributions of potential replication origins (grey ribbons) are relatively even in the TAD. The TAD comprises several chromatin loops (blue) organized by CTCF and cohesin at the loop anchors (green rings). PCNA clusters (yellow balls) surrounding the TAD are bound to the nuclear matrix (hazed light blue straws). b-c, With transcription proceeding, the chromatin loops undergo structural re-organization along with chromatin domain decompaction in the G1 phase, exposing a subset of the origins to the periphery of the TAD (pink ribbons). Note that the origin at the sequence boundary of the TAD remains at the TAD periphery in the G1 phase. These peripheral origins are more accessible to the surrounding PCNA clusters and thus become active origins for the initiation of DNA replication at the periphery of the TAD. The areas inside the black squares in $\mathbf{a}$ and $\mathbf{b}$ are shown at higher magnification above. 


\section{Figures and Figure Legends}

a

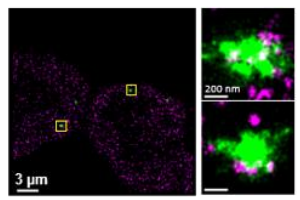

EdU 10 min TAD1

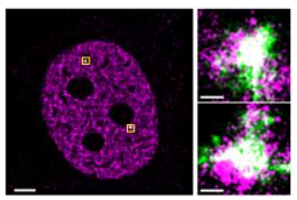

EdU 60 min TAD1

b

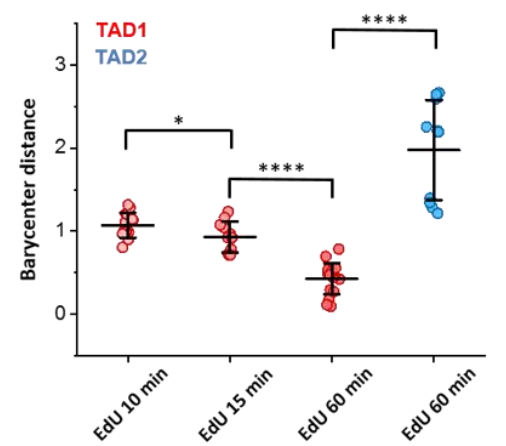

C

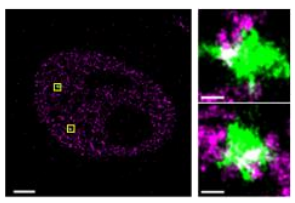

EdU 15 min TAD1

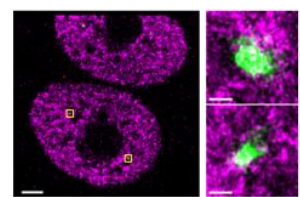

EdU 60 min TAD2

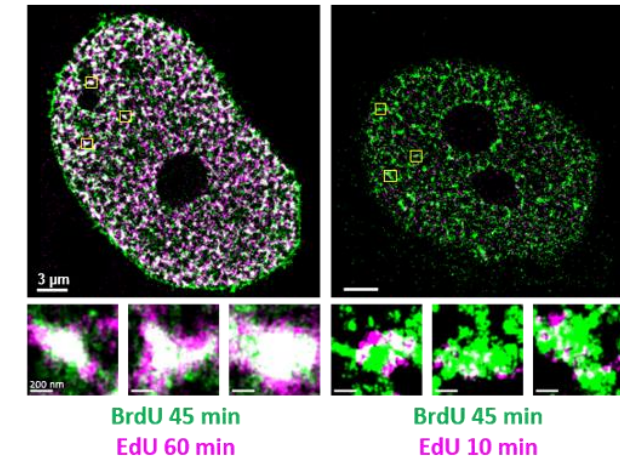

d

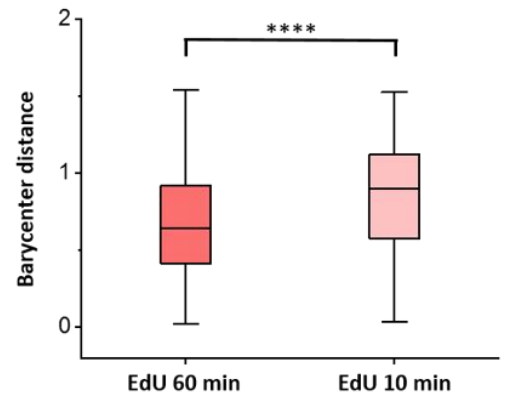

Figure 1. Super-resolution imaging of RFi and TADs in the $\mathbf{S}$ phase. a, Representative STORM images of TAD1 and TAD2 labeled by Oligopaint probes (green) and RFi labeled metabolically for different durations (purple) (Methods). TAD1 and TAD2 were chosen based on the replication timing profile and Hi-C interaction heatmap of HeLa cells (Appendix Figure S1). TAD1: an early replicating domain (Chr1:16911932-17714928). TAD2: a middle replicating domain (Chr1:17722716-18846245). Metabolic labeling of DNA replication was performed by supplying EdU to the cell upon release into the $\mathrm{S}$ phase for $10 \mathrm{~min}, 15 \mathrm{~min}$, and $60 \mathrm{~min}$ (purple). The areas inside the yellow squares are shown at higher magnification to the right of each nucleus. Portions of the two signals that overlap are shown in white. b, Barycenter distances between the TAD and its spatially associated RFi (Methods) in a. Horizontal lines and error bars represent the mean values \pm s.d. of three or more independent biological replicates ( $n=16$ cells). $\mathbf{c}$, Representative STORM images of RFi labeled metabolically for different durations in two consecutive cell cycles. Consecutive metabolic labeling of DNA replication was performed by supplying BrdU (green) to the cell upon release into the $S$ phase in the first cell cycle, followed by supplying EdU (purple) to the cell upon release into the $\mathrm{S}$ phase in the second cell cycle (for different durations). The areas inside the yellow squares are shown at higher magnification below each nucleus. d, Box plot of barycenter distances between BrdU and EdU labeled RFi in $\mathbf{c}$ (data were pooled from $\mathrm{n}=10$ cells). Center line, median; box limits, $25 \%$ and $75 \%$ of the entire population; whiskers, observations within $1.5 \times$ the interquartile range of the box limits. Significance was analyzed by un-paired two sample parametric t test. $* * * * \mathrm{P}<0.0001,{ }^{* * *} \mathrm{P}<0.0005, * * \mathrm{P}<0.01, * \mathrm{P}<0.05$, N.S.: not significant. 3D results are shown in Fig. S2\&S5. 

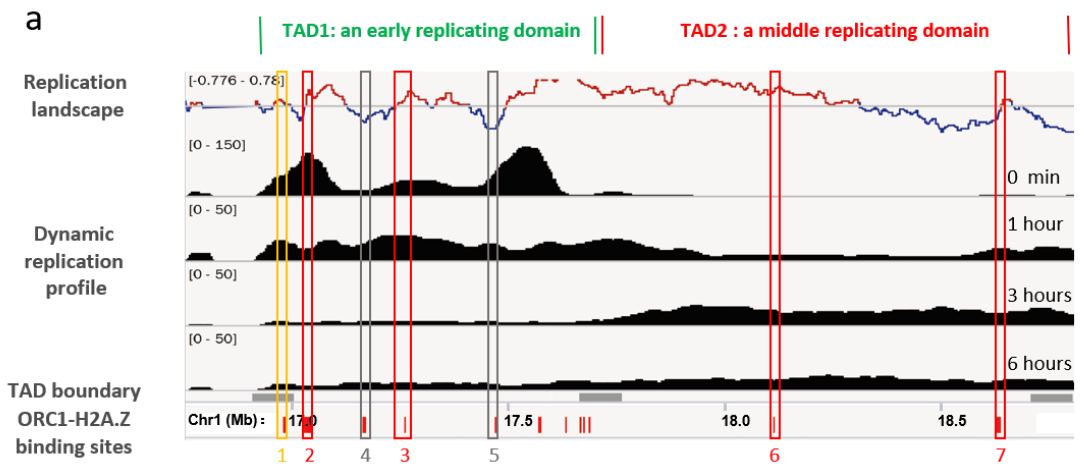

b
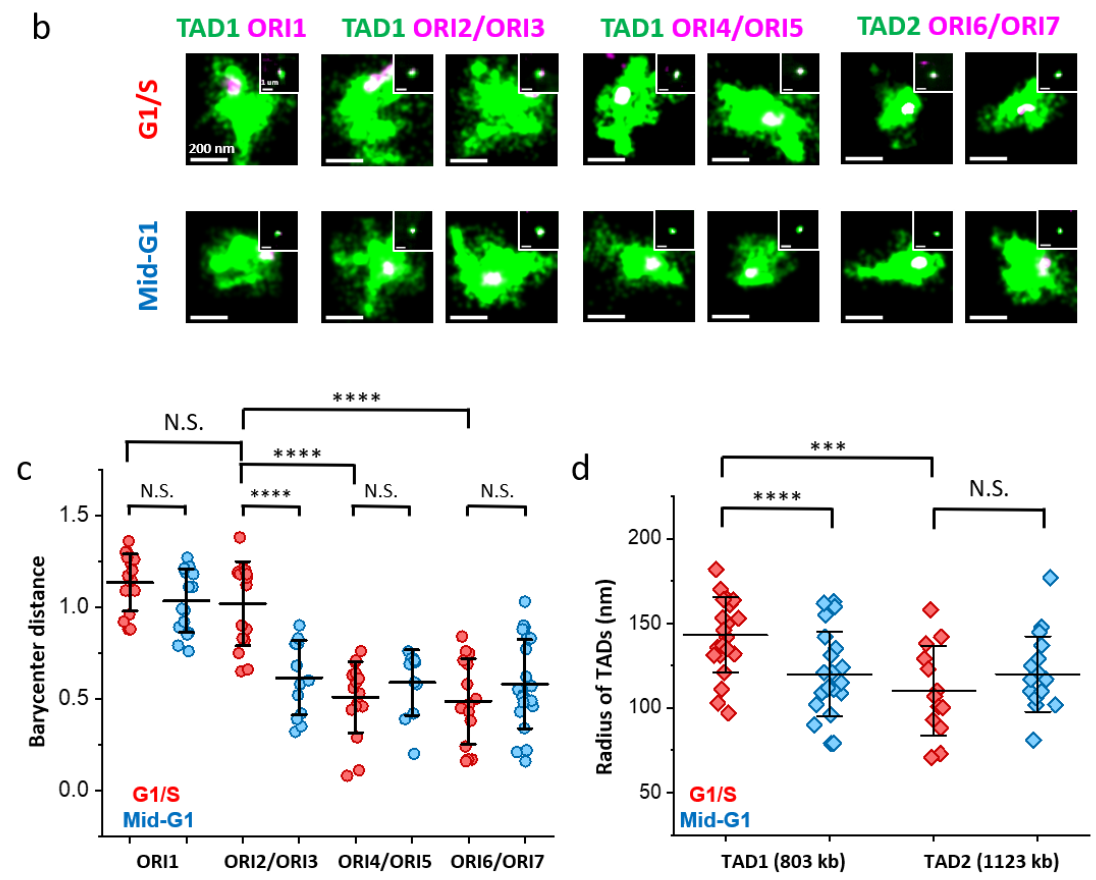

Figure 2. Spatial distribution of replication origins relative to the TADs in the G1 and G1/S phases. a, A scheme of replication in TAD1 and TAD2. The top profile represents the replication landscape obtained by OK-seq. (-0.776-0.78) was the threshold of OK-seq [81]. The middle black peaks represent the dynamic replication profile, which was obtained by 10 -min BrdU pulse labeling at $0 \mathrm{~min}, 30 \mathrm{~min}, 3$ hours, and 6 hours into the S phase. $(0-50)$ or $(0-150)$ is the range of normalized BrdU-seq data. The grey bars represent the TAD boundaries in the RDs. The small red bars at the bottom represent the ORC1 and $\mathrm{H} 2 \mathrm{~A} . \mathrm{Z}$ binding sites indicating the potential replication origins. Representative active and dormant replication origins defined by the BrdU-seq data and the OK-seq profile are marked with vertical rectangles. Yellow rectangle: active replication origin (ORI1) at the TAD boundary. Red rectangles: active replication origins in TAD1 (ORI2 and ORI3) and TAD2 (ORI6 and ORI7). Black rectangles: dormant replication origins in TAD1 (ORI4 and ORI5). b, Representative STORM images of TADs (green) and their origins (purple) labeled by FISH with oligoprobes in the G1 and G1/S phases. Upper, TADs and origins labeled at the G1/S transition. Lower, TADs and origins labeled at approximately 5 hours into the G1 phase. Portions of the two signals that overlap are shown in white. The corresponding conventional images are shown in the inset. $\mathbf{c}$, Barycenter distances between origins and TADs in $\mathbf{b}(\mathrm{n} \geq 10$ cells). d, Radii of TAD1 and TAD2 in the $\mathrm{G} 1$ or $\mathrm{G} 1 / \mathrm{S}$ phase ( $\mathrm{n} \geq 10$ cells). For lines and statistics in $\mathbf{c}$ and $\mathbf{d}$ see the description in the legend of Figure 1.3D results are shown in Fig. S9. 
a

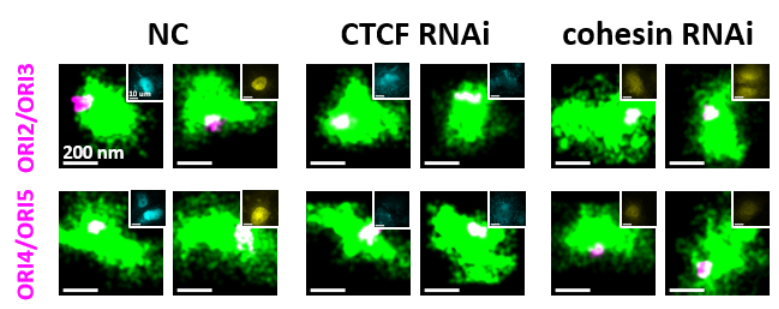

b

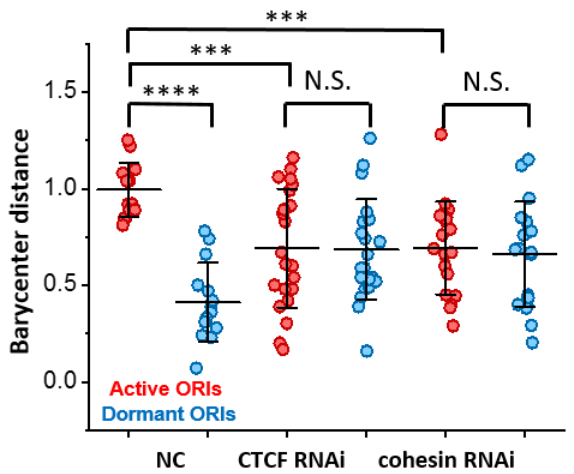

C

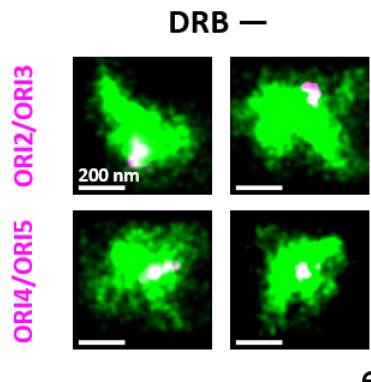

d

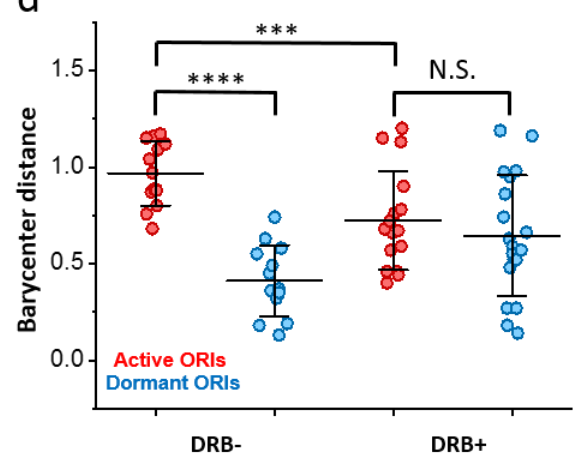

e

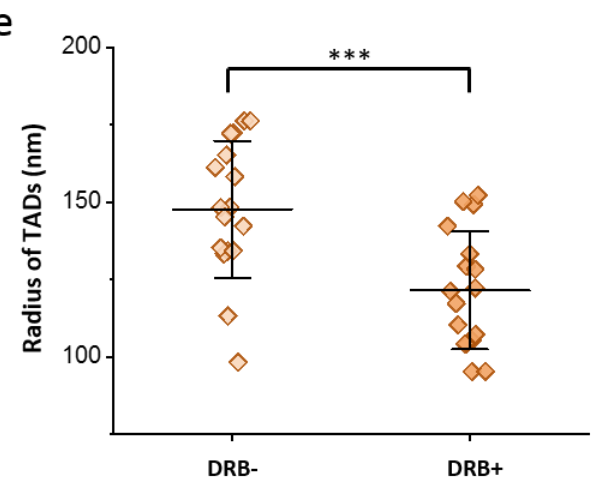

Figure 3. The spatial distribution of replication origins within the TADs at the G1/S transition is dependent on CTCF, cohesin and transcription. a, Representative STORM images of origins (purple) in TAD1 (green) after treatment of cells with the indicated siRNAs. Conventional images indicate the concentration of CTCF (cyan) or cohesin (yellow) in the nucleus. b, Barycenter distances between active or dormant origins in TAD1 after treatment of cells with the indicated siRNAs. Portions of the two signals that overlap are shown in white. c, Representative STORM images of origins (purple) in TAD1 (green). Left: no DRB. Right: with DRB. d, Barycenter distances between active/dormant origins and TAD1 with or without DRB treatment. e, Radius of TAD1 treated with or without DRB. For lines and statistics in $\mathbf{b}, \mathbf{d}$, and $\mathbf{e}$ see the description in the legend of Figure 1 ( $\mathrm{n} \geq 10$ cells). 3D results are shown in Fig. S10. 

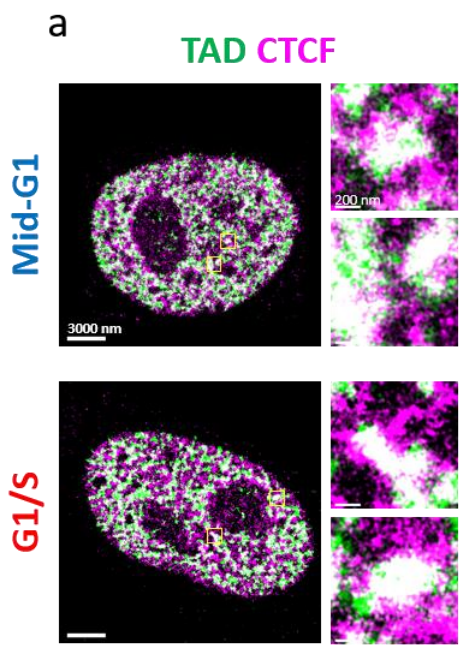

b
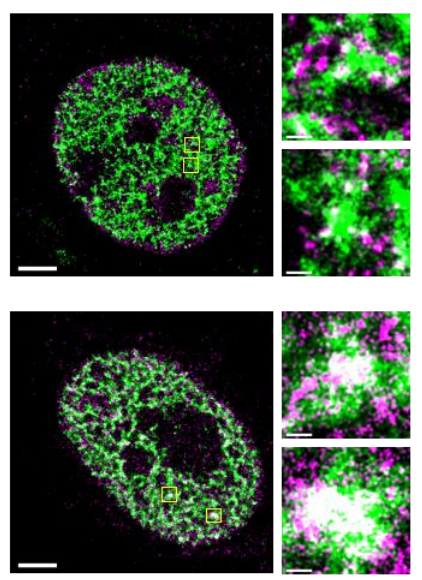

$\mathrm{C}$

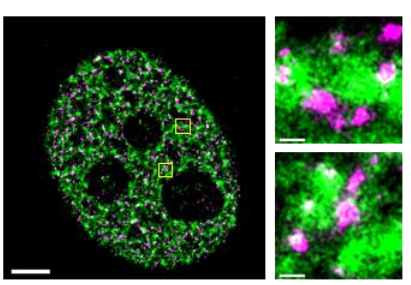

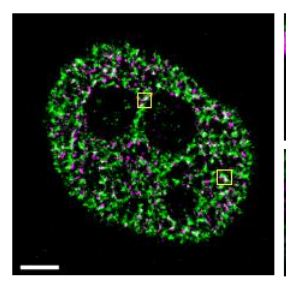

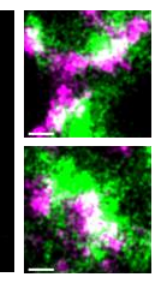

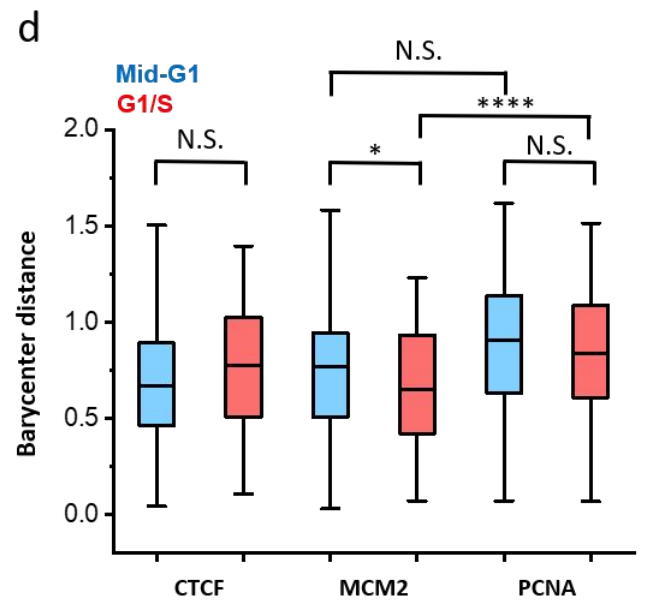

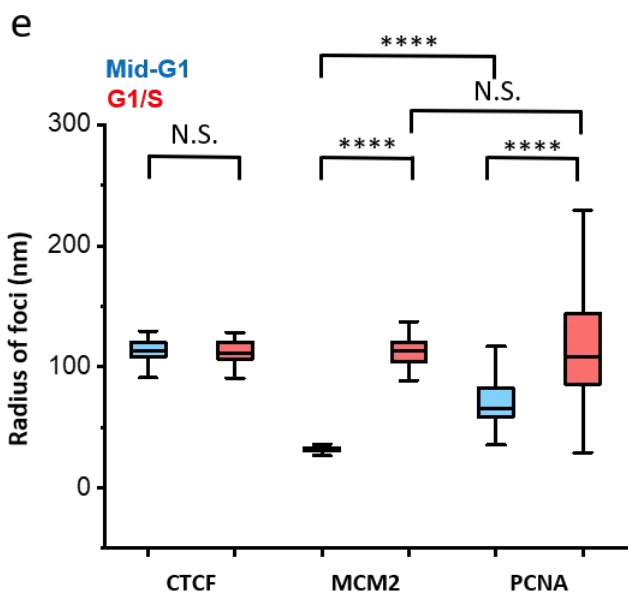

57 Figure 4. Spatial distributions of CTCF, MCM2 and PCNA relative to the early replicating 58 TADs in the G1 and G1/S phases. a-c, Representative STORM images of CTCF, MCM2 and 59 PCNA labeled by immunostaining (purple) and metabolically labeled TADs (green). Cells were 60 fixed and labeled in the mid-G1 phase (upper) or G1/S phase (lower). The areas inside the yellow 61 squares are shown at higher magnification next to each nucleus. Portions of the two signals that overlap are shown in white. d, Barycenter distances between CTCF, MCM2 or PCNA with the TADs in the mid-G1 phase or G1/S phase. e, Radii of CTCF, MCM2 or PCNA foci in the mid-G1 phase or $\mathrm{G} 1 / \mathrm{S}$ phase. For lines and statistics in $\mathbf{d}$ and $\mathbf{e}$ see the description in the legend of Figure $1(\mathrm{n}=10$ cells). 

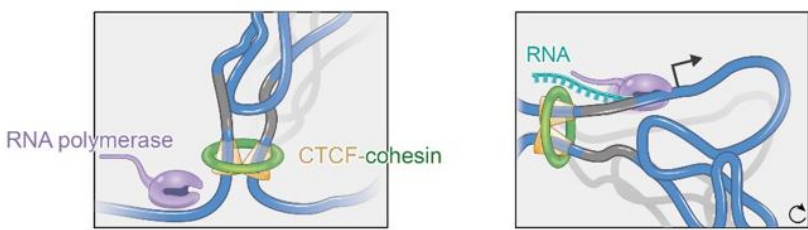

b

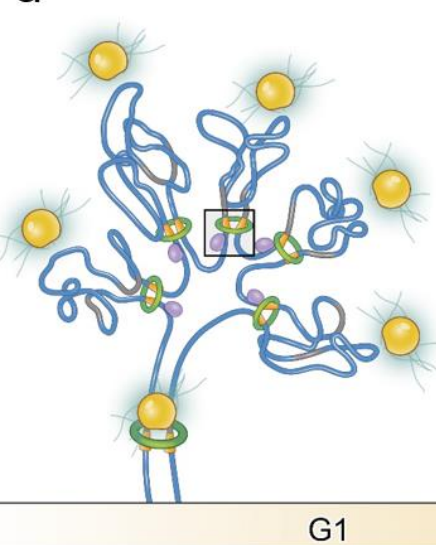

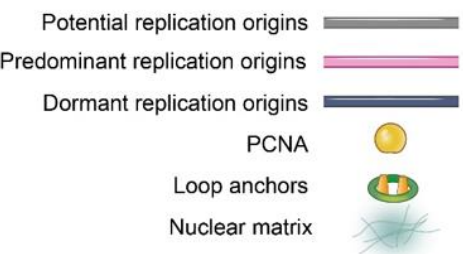

C

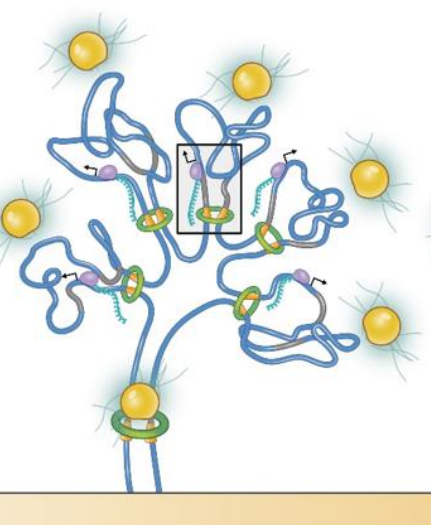

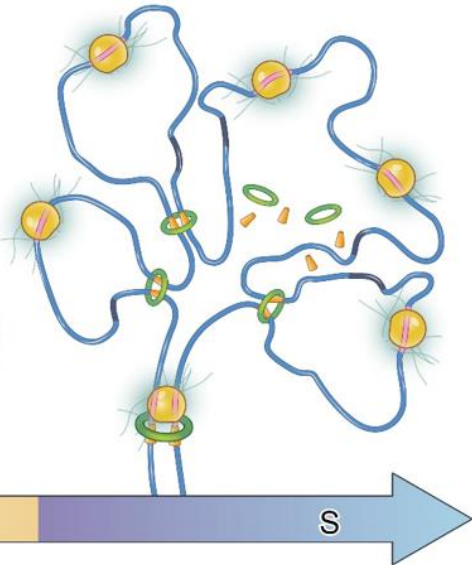

Figure 5. "Chromatin Re-organization Induced Selective Initiation" (CRISI) model for selective initiation of DNA replication origins. a, In the early G1 phase, the spatial distributions of potential replication origins (grey ribbons) are relatively even in the TAD. The TAD comprises several chromatin loops (blue) organized by CTCF and cohesin at the loop anchors (green rings). PCNA clusters (yellow balls) surrounding the TAD are bound to the nuclear matrix (hazed light blue straws). b-c, With transcription proceeding, the chromatin loops undergo structural re-organization along with chromatin domain decompaction in the G1 phase, exposing a subset of the origins to the periphery of the TAD (pink ribbons). Note that the origin at the sequence boundary of the TAD remains at the TAD periphery in the G1 phase. These peripheral origins are more accessible to the magnification above. 
a

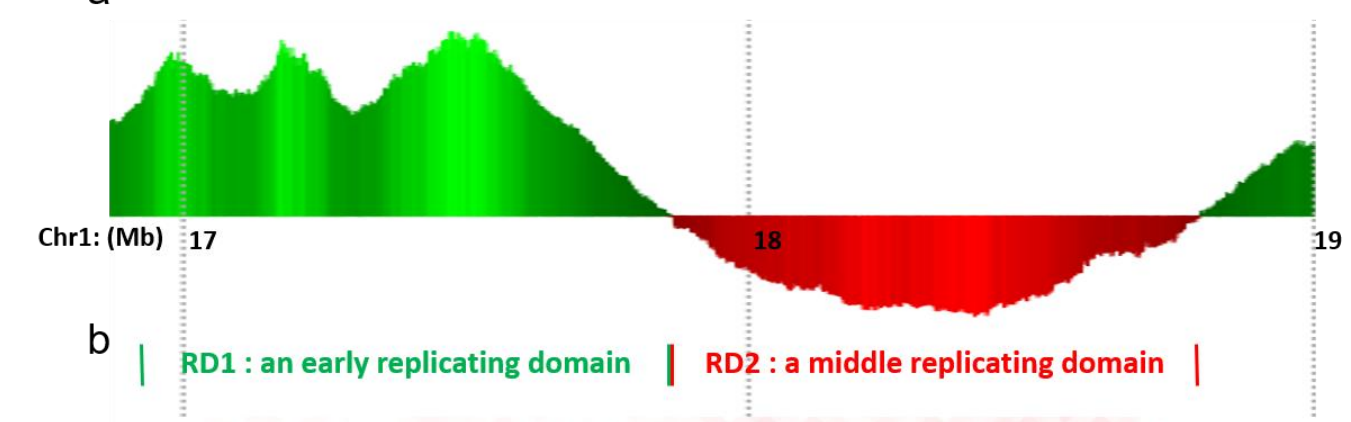

Appendix Figure S1 Identification and histone modifications of two TADs from the replication iming profile and Hi-C interaction heatmap of HeLa cells. a-b, Depiction of the replication timing profile (green peaks for early RDs and red peaks for middle or late RDs) and Hi-C interaction heatmap in the same genomic region of chromosome 1. The replication timing profile was obtained

from the Replication Domain Genome Browser of the Gilbert lab

89 (https://www2.replicationdomain.com/ genome browser). The Hi-C interaction heatmap was

90 obtained from ENCSR693GXU. Grey bars: TAD boundaries. (Methods) Two TADs were selected.

91 TAD1: an early replicating domain (Chr1:16911932-17714928). TAD2: a middle replicating

92 domain (Chr1:17722716-18846245). c, Profiles of histone modifications are from public data

93 hubs (ENCODE data portal) of WashU Epigenome Browser. 
a

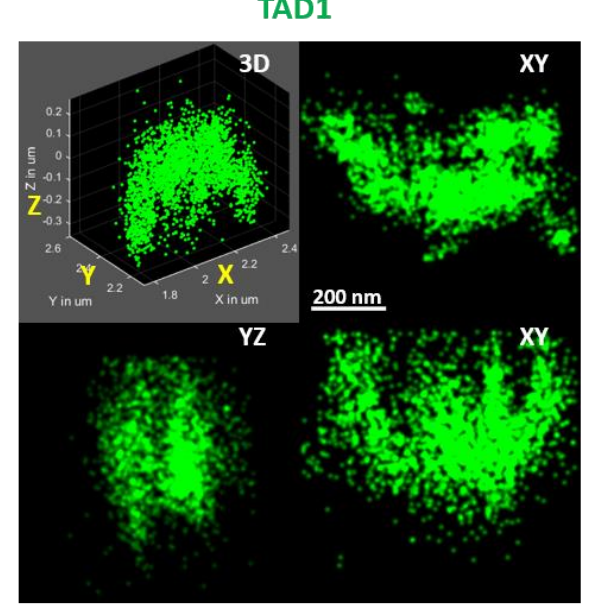

TAD2

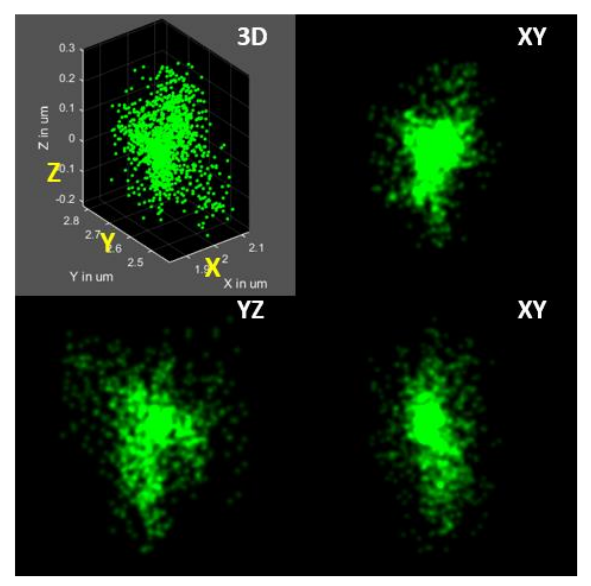

b

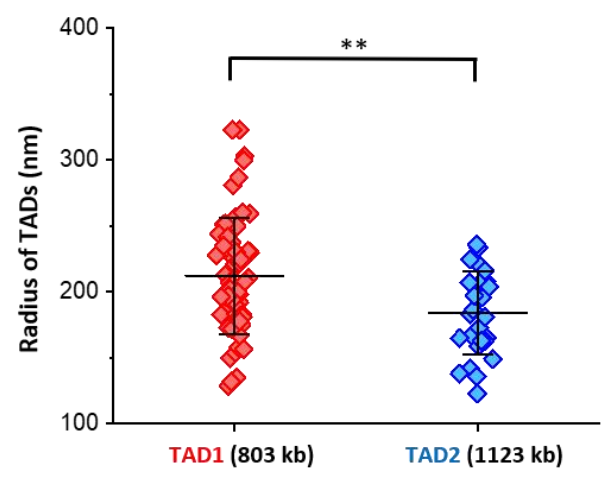

96

Appendix Figure S2. 3D visualization and radii of TAD1 and TAD2. The definitions and labeling procedures of TADs and origins are identical with those in Figure 2. a, Representative 3D STORM images of TADs. One 3D presentation with 3 projected images. $\mathbf{b}, 3 \mathrm{D}$ radius of gyration of TAD1 and TAD2. For lines and statistics in $\mathbf{b}$ see the description in the legend of Figure 1 ( $\mathrm{n} \geq 20$ cells). 
a
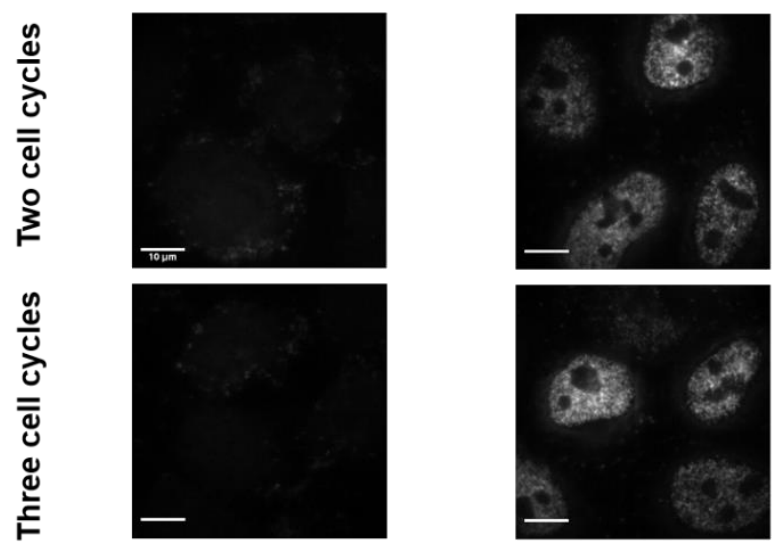

no release

released for $15 \mathrm{~min}$

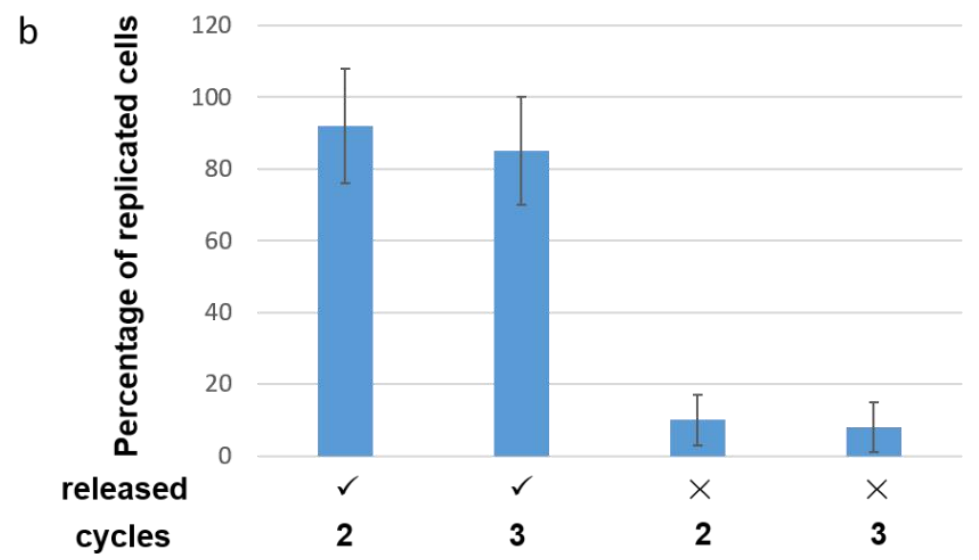

Appendix Figure S3. Quantification of cell synchronization by EdU labeling. To make sure the cells were successfully synchronized and the synchronization procedure minimally impacts the growth and morphology of cells, we imaged and analyzed the cells synchronized for two or three cell cycles. a, after two (upper) or three (lower) cycles of synchronization, cells were synchronized to the G1/S transition. EdU were added when the synchronized cells were released (right) for 15 min or were not released (left). b, percentage of the replicated cells in a. Replicated cells were defined by the three folds of the fluorescence of the nucleus to the background ( 3 replicates, 200 cells for each group). EdU labeling showed more than $80 \%$ cells entering the $\mathrm{S}$ phase, similar with 
a

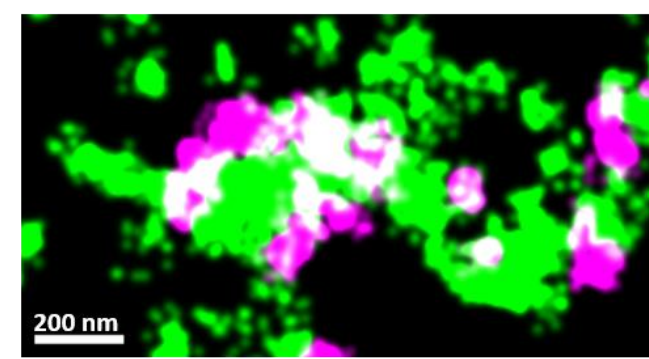

d

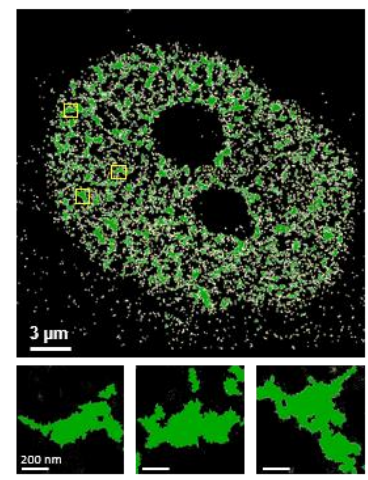

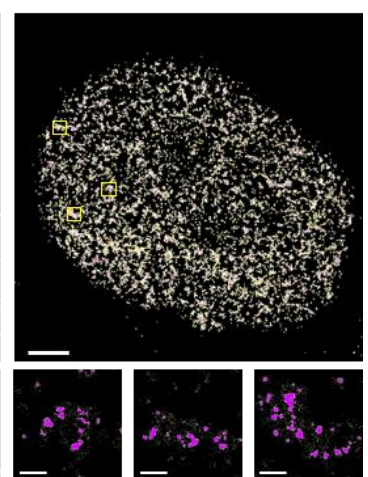

b
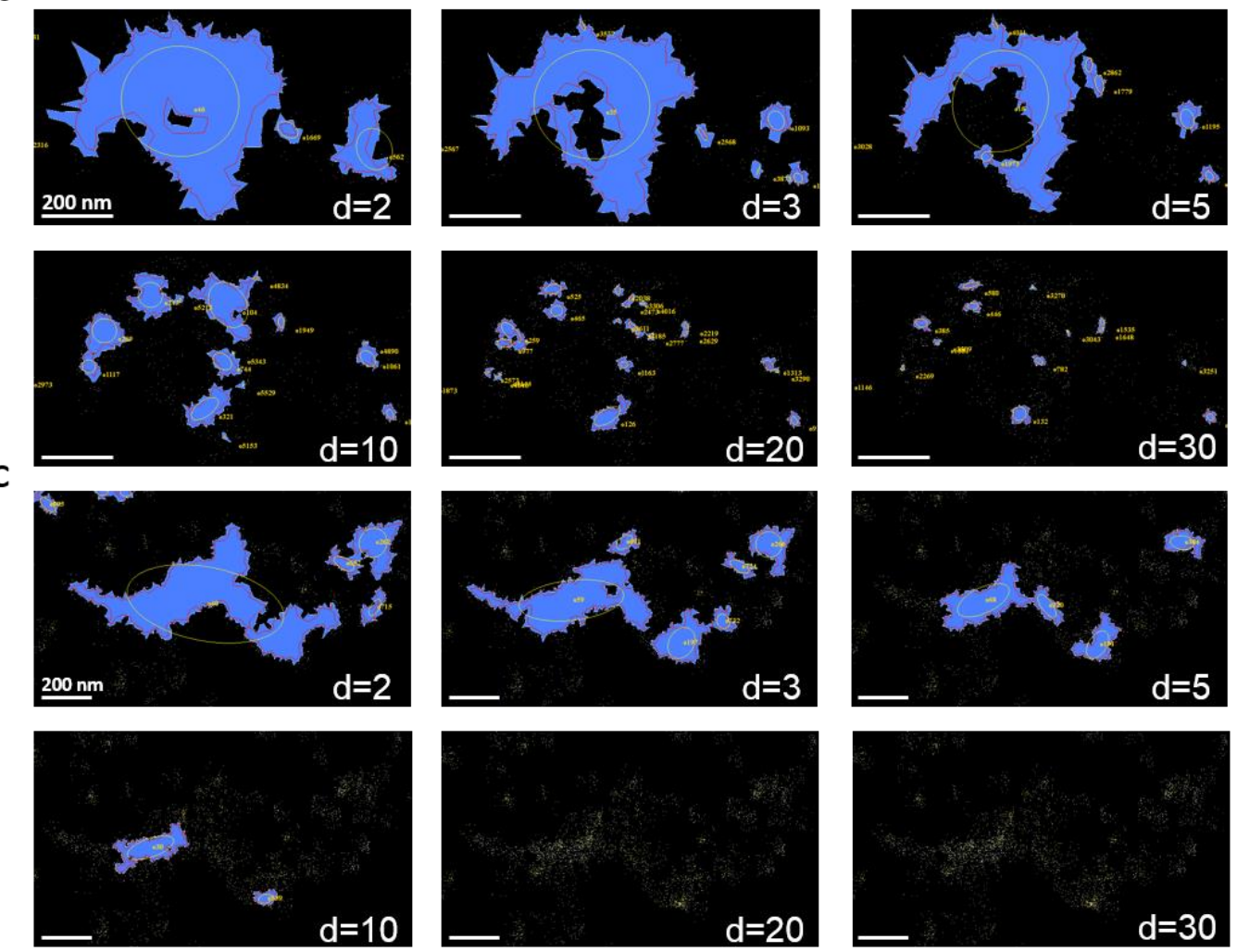

Appendix Figure S4. Quantification of the density factor in SR-Tesseler analysis. a, Dual-color RM imaging of TADs (also early replicating domains, green) and origins (purple) as in Fig. 1c. c, Analysis of TADs and origins by SR-Tesseler with density factors from 2 to 30 . bo, Origins close to each other in a TAD cannot be separated when the density factor is set from 2 to 10 . Origins are too small or even dismissed when the density factor is set to 30 . When the density factor was set to 20 , approximately 5,000 origins were clearly defined at the beginning of the $\mathrm{S}$ phase in one cell, similar with a previous report [2]. c, TADs close to each other cannot be separated when the density tor is set to 2. TADs are too small or even dismissed when the density factor is set from 5 to 30 . 128 a previous report[3]. d, TADs (green) and origins (purple) identified by SR-Tesseler from the 129 STORM images using a density factor of 3 for TADs and a density factor of 20 for origins. The areas inside the yellow squares are shown at higher magnification below each nucleus. 
a

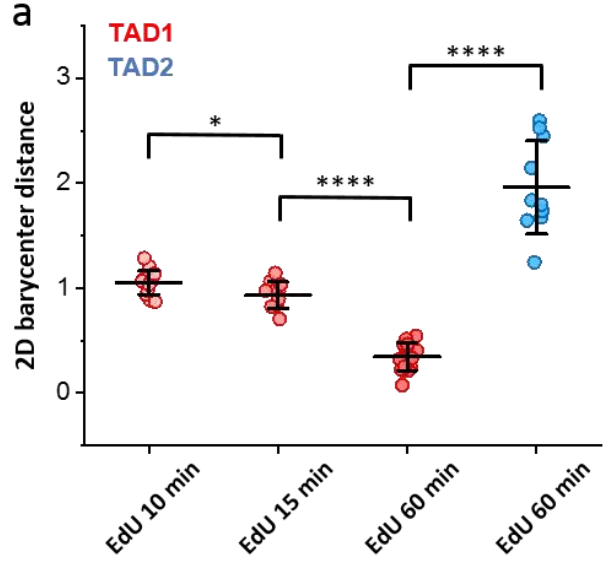

C

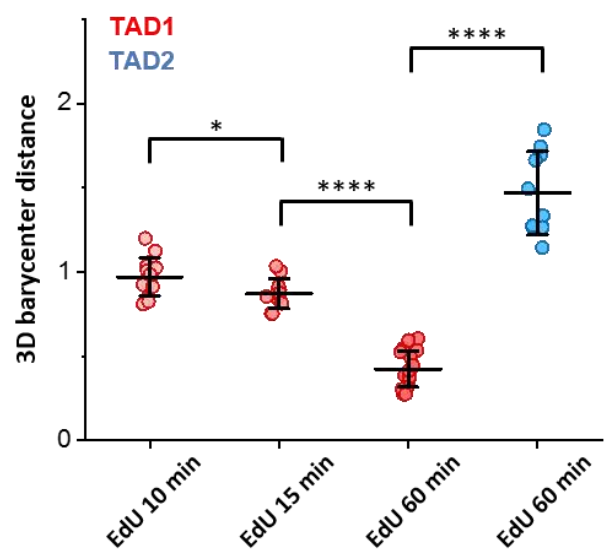

b

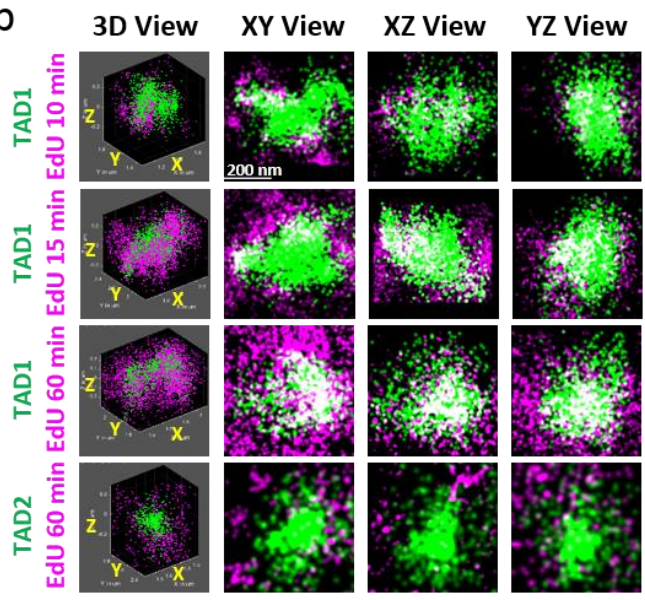

d

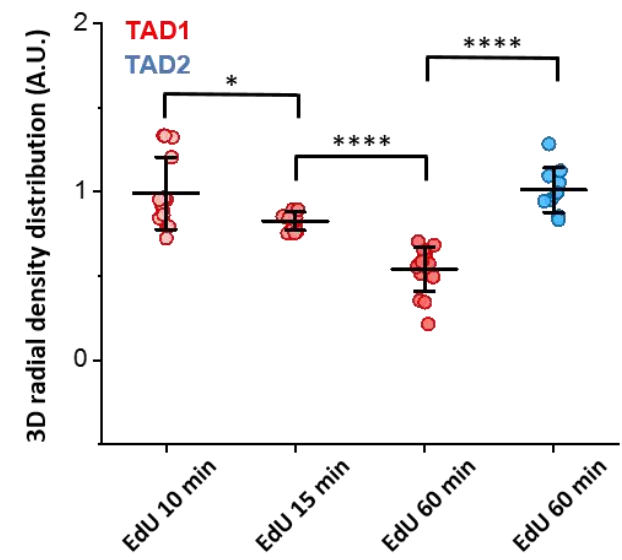

Appendix Figure S5. Replication patterns of TADs in the S phase as determined by DBSCAN. a, 2D barycenter distances between the TADs and their spatially associated RFi as determined by DBSCAN (Methods) in Figure 1a. b, Representative 3D STORM images of TAD1 and TAD2 labeled by Oligopaint probes (green) and RFi labeled metabolically for different durations (purple). Metabolic labeling of DNA replication was performed by supplying EdU to the cells upon release into the $\mathrm{S}$ phase for $10 \mathrm{~min}, 15 \mathrm{~min}$, and $60 \mathrm{~min}$ (purple). c, 3D barycenter distances between the TADs and their spatially associated RFi as determined by DBSCAN in b. d, Radial density distribution of the RFi in TADs as determined by DBSCAN (Methods) in b. For lines and statistics in $\mathbf{a}, \mathbf{c}$, and $\mathbf{d}$ see the description in the legend of Figure 1 ( $\mathrm{n} \geq 10$ cells). 
a

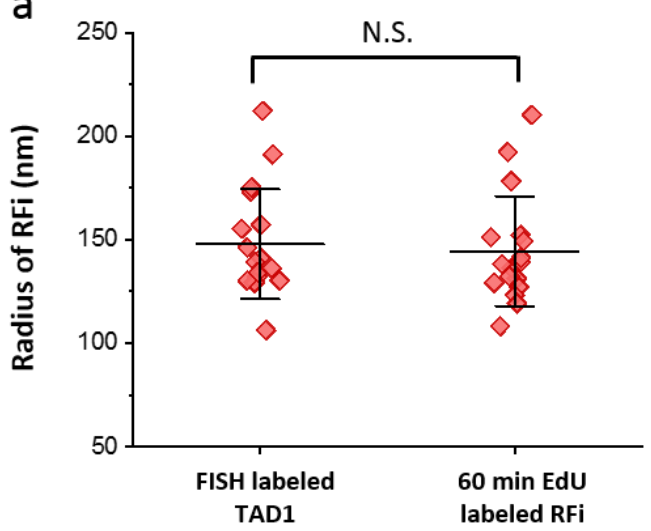

C

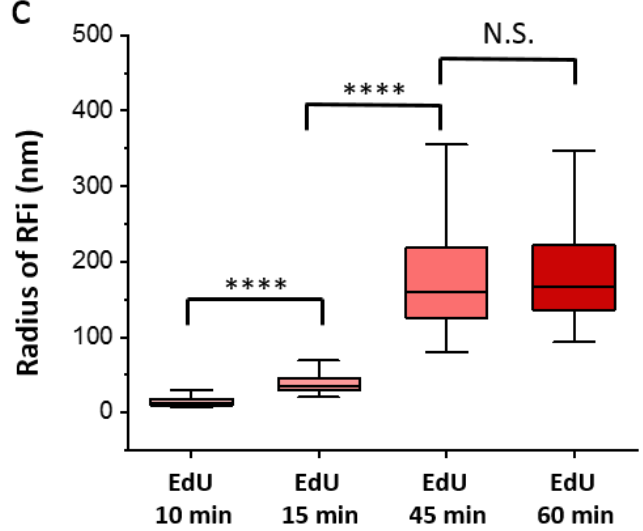

b

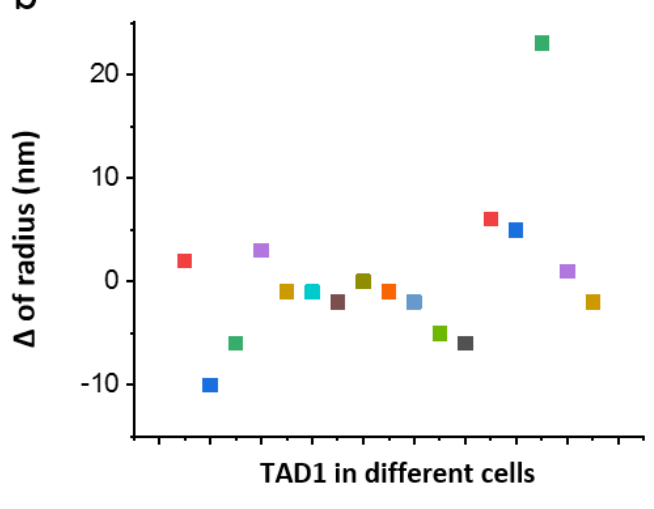

d

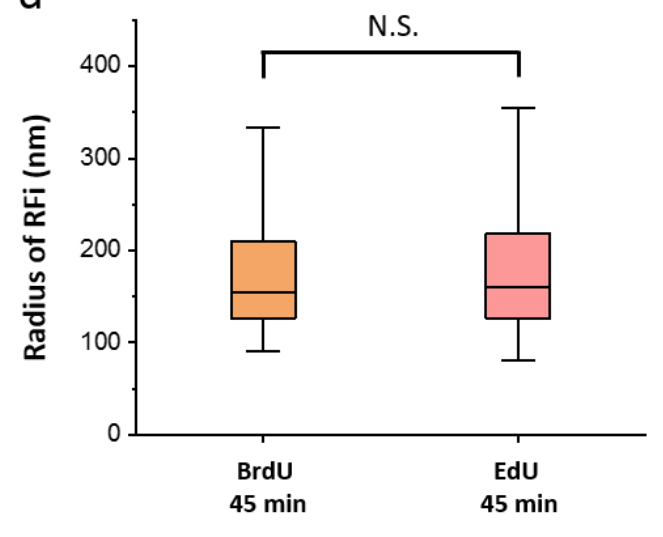

147 Appendix Figure S6. Quantitative characterization of metabolically labeled RFi. a \& b, 148 Comparison of FISH-labeled TAD1 and co-localized 60-min EdU labeled RFi. a, Radii of FISH149 labeled TAD1 and 60-min EdU labeled RFi. b, Changes in radius between FISH-labeled TAD1 and 150 its corresponding 60-min EdU labeled RFi in the same cell. Different colors represent different cells. $151 \mathrm{c}$, Radii of RFi labeled for $10 \mathrm{~min}, 15 \mathrm{~min}, 45 \mathrm{~min}$ and $60 \mathrm{~min}$ upon release into the $\mathrm{S}$ phase. d, 152 Radii of RFi labeled for 45 min by BrdU (yellow) or EdU (pink) at the beginning of the S phase. 153 For lines and statistics in $\mathbf{a}, \mathbf{c}$, and $\mathbf{d}$ see the description in the legend of Figure 1 ( $\mathrm{n}=10$ cells). 
a

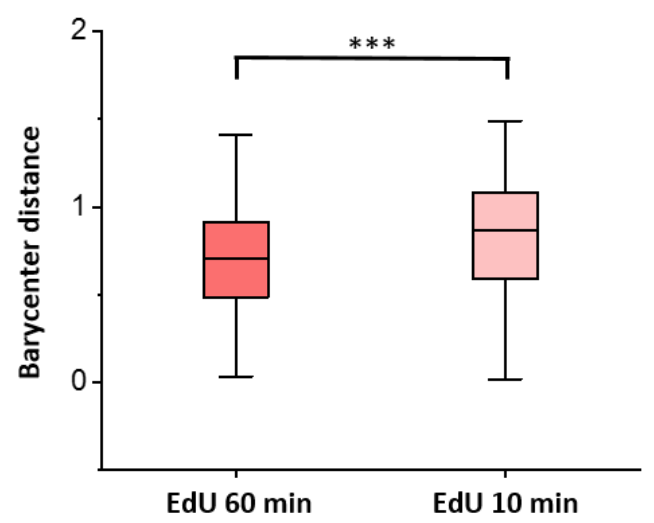

b

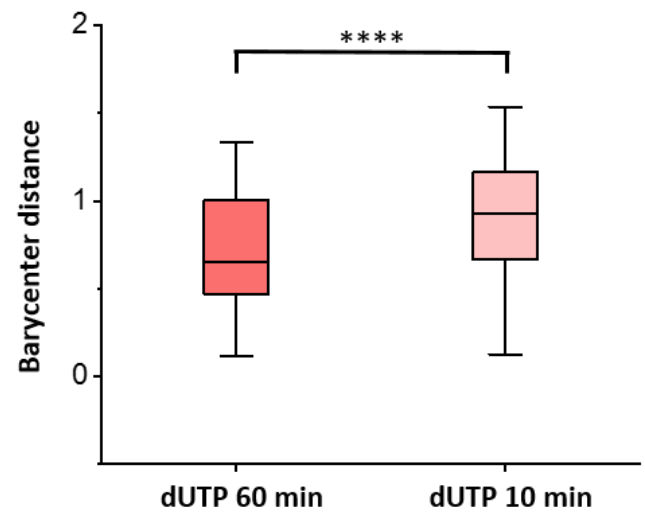

158 Appendix Figure S7. Comparison of RFi labeled by different metabolic labeling methods and 159 for different durations. a, Box plot of barycenter distances between BrdU-labeled RFi and EdU160 labeled RFi. BrdU was supplied for $45 \mathrm{~min}$ upon release into the $\mathrm{S}$ phase, whereas EdU was supplied 161 for 10 or $60 \mathrm{~min}$. b, Box plot of barycenter distances between EdU-labeled RFi and dUTP-atto550 162 labeled RFi. EdU was supplied for 45 min upon release into the $\mathrm{S}$ phase, whereas dUTP was supplied 163 for 10 or $60 \mathrm{~min}$. For lines and statistics in $\mathbf{a}$ and $\mathbf{b}$ see the description in the legend of Figure 1 ( $\mathrm{n}$ $164=10$ cells). 
171 Appendix Figure S8. Co-localization of replication origins with CTCF-cohesin binding sites.

172 Histograms of distance between $\mathrm{CTCF}$-cohesin binding sites and replication origins are presented

173 in solid lines; those for randomly-selected sites are presented by dotted line. TBA (TAD boundary

174 active origins): red lines; NBA (Non-TAD boundary active origins): yellow lines; D (dormant 175 replication origins): grey lines. Center dashed line is the sites with overlapped binding of CTCF and 176 cohesin.

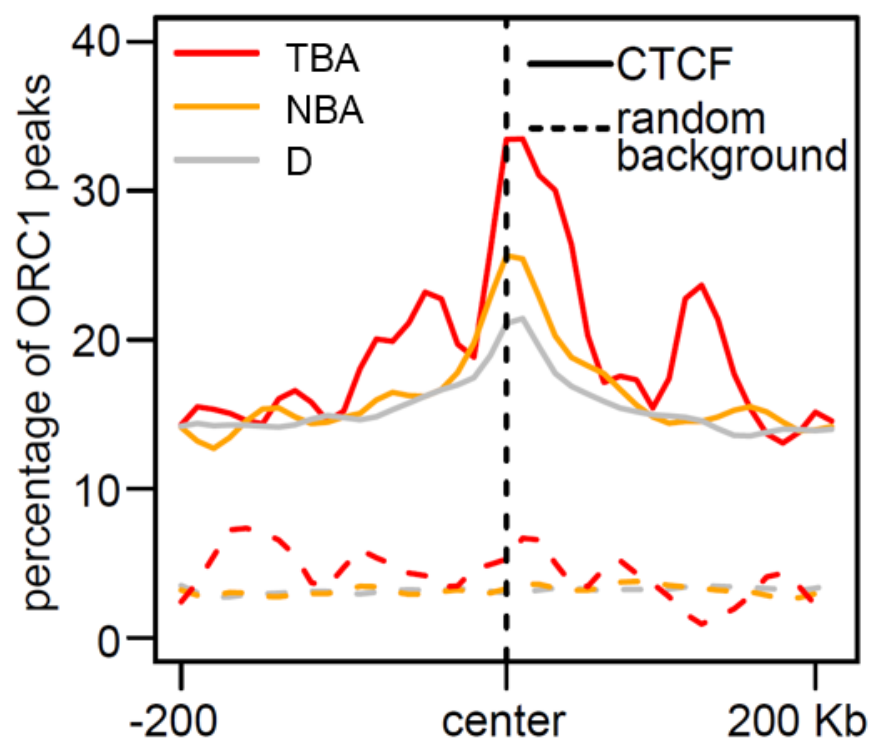


a
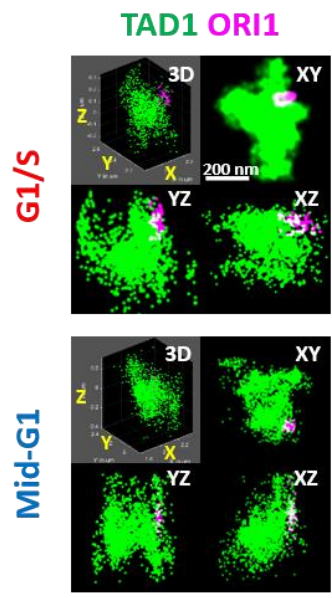

\section{TAD1 ORI2}

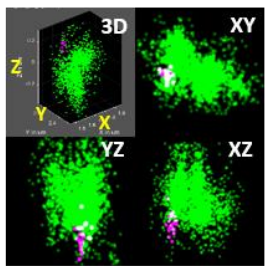

TAD1 ORI4
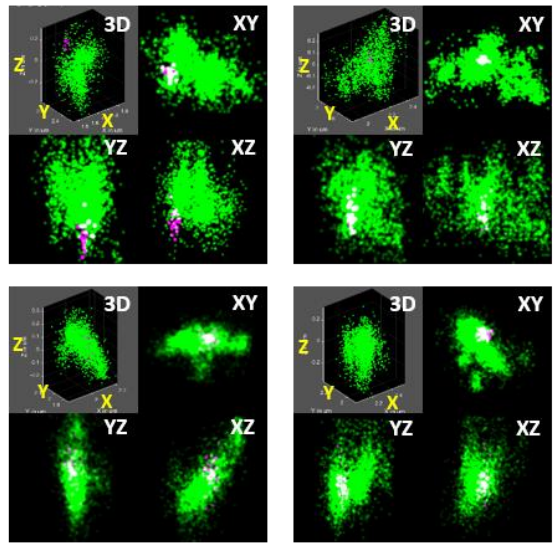

TAD2 ORI6
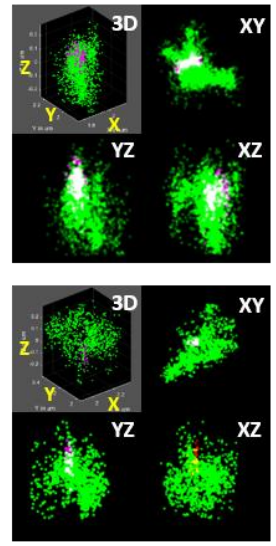
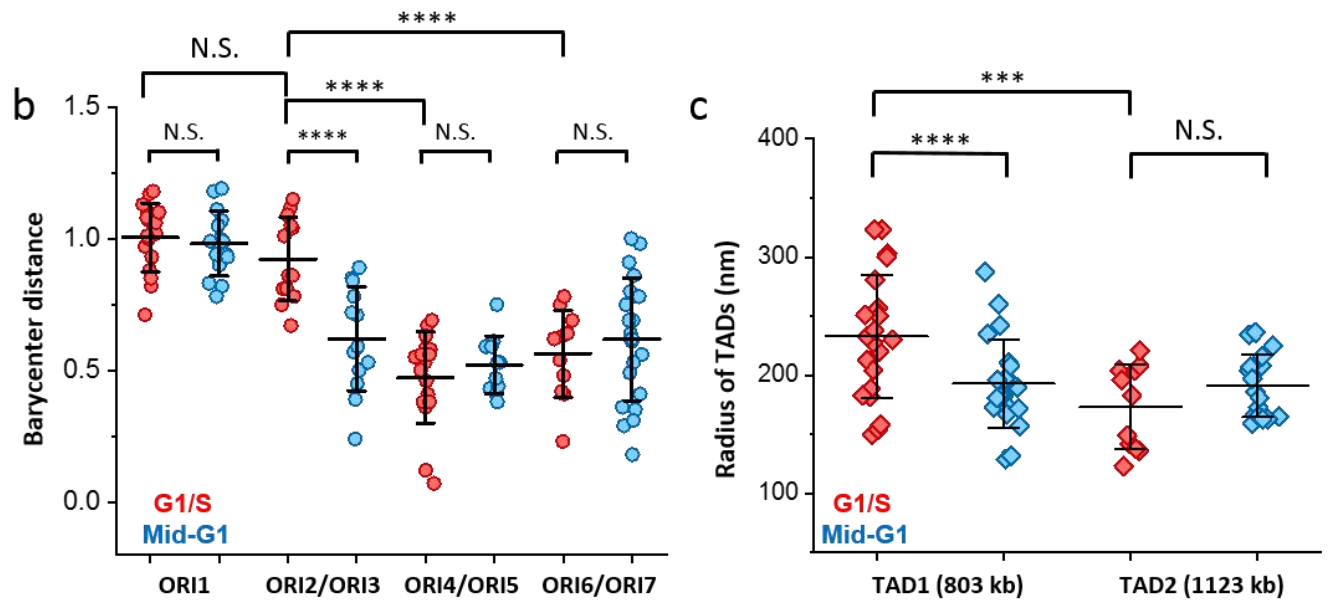

Appendix Figure S9. 3D distribution of replication origins in TADs in the G1 and G1/S phase.

188 Representative 3D STORM images of TADs (green) and their origins (purple) in the G1 and G1/S 189 phases. Upper row: TADs and origins labeled at the G1/S transition. Lower row: TADs and origins 190 labeled approximately 5 hours into the G1 phase. b, 3D Barycenter distances between all 7 origins 191 and the 2 related TADs in a. c, 3D radius of gyration of TAD1 and TAD2 in the G1 and G1/S phases. 192 For lines and statistics in $\mathbf{b}$ and $\mathbf{c}$ see the description in the legend of Figure 1 ( $n \geq 10$ cells). 

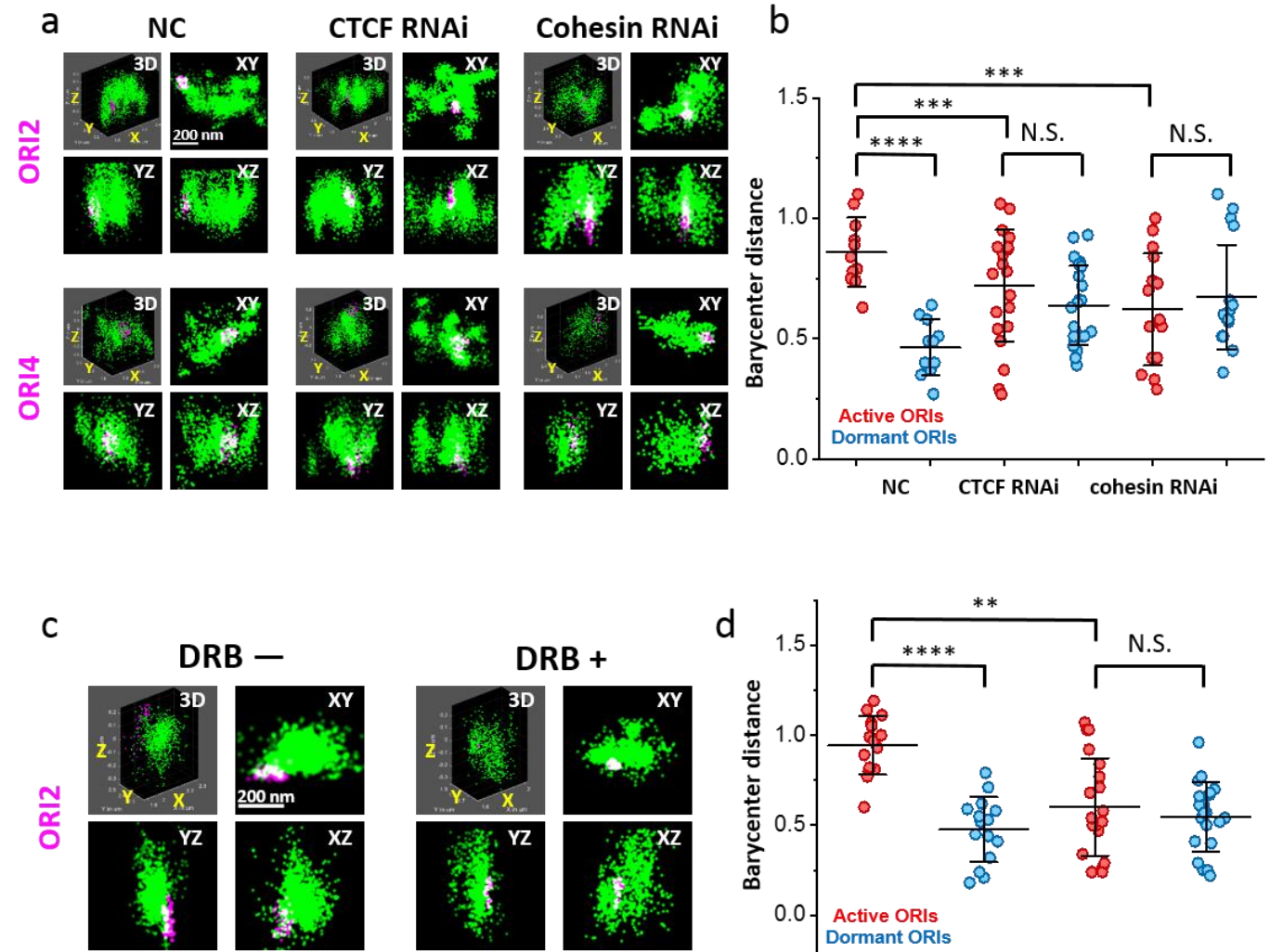

DRB +
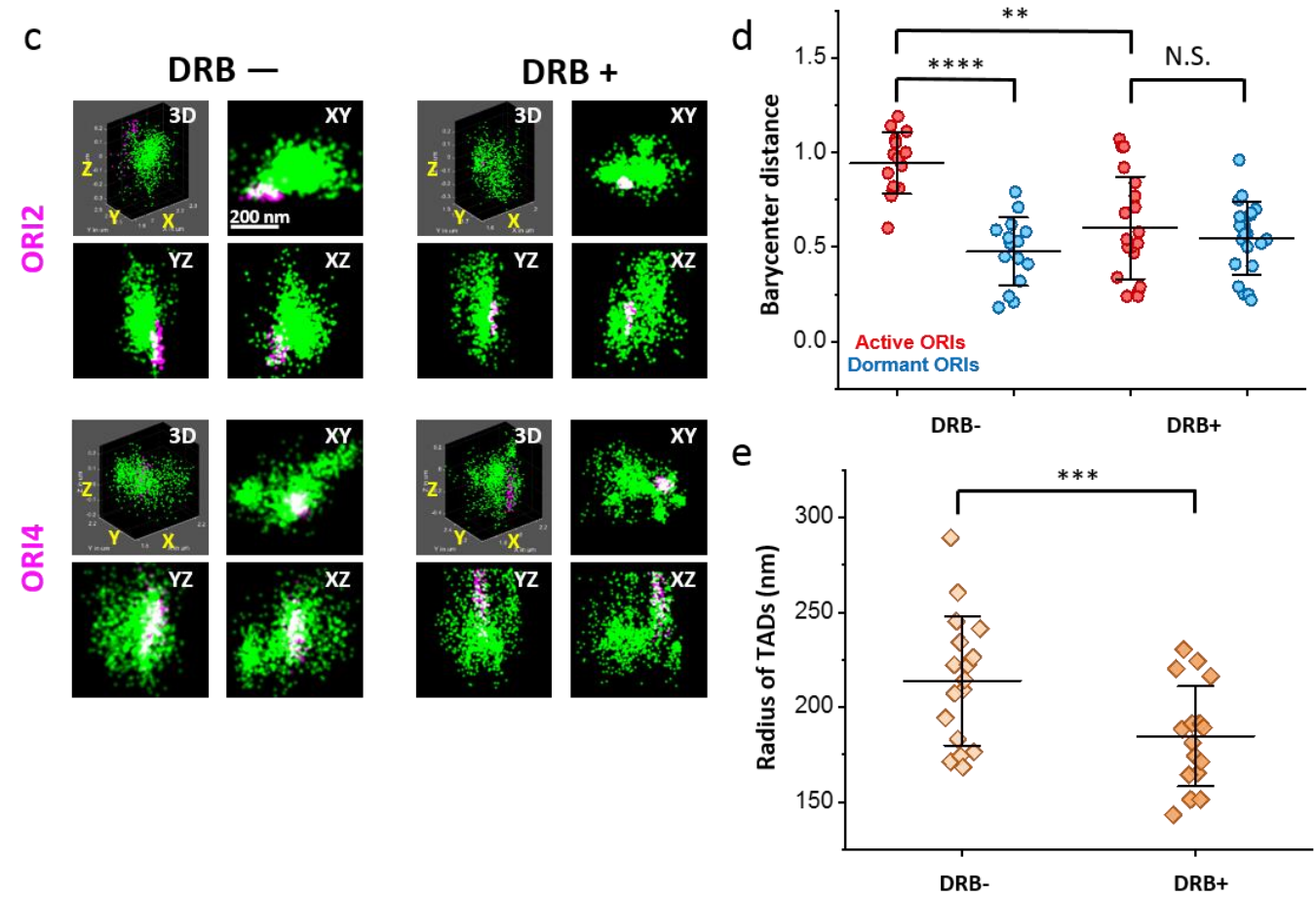

Appendix Figure S10. 3D Distribution of replication origins in TAD1 with transcription elongation inhibition or down-regulation of CTCF or cohesin. a, Representative 3D STORM images of origins (purple) in TAD1 (green) after treatment of cells with the indicated siRNAs. b, 3D barycenter distances between active (ORI2 and ORI3) or dormant (ORI4 and ORI5) origins in TAD1 after treatment of cells with the indicated siRNAs as in a. c, Representative 3D STORM images of origins (purple) in TAD1 (green). Restricted by the space, only ORI2 and ORI4 are shown. Left: no DRB. Right: with DRB. d, 3D barycenter distances between active (ORI2 and ORI3) and dormant (ORI4 and ORI5) origins in TAD1 with or without DRB treatments. e, 3D radius of gyration of TAD1 treated with or without DRB. For lines and statistics in $\mathbf{b}$, d, and $\mathbf{e}$ see the description in the legend of Figure 1 ( $n \geq 10$ cells). 
Appendix Figure S11. Transcription of origin-associated genes in the G1 phase. The expression data of the genes in TAD1 and TAD2 were obtained from the RNA-seq data set in the NCBI Gene Expression Omnibus (GEO; http://www.ncbi.nlm.nih.gov/geo/) under accession number GSE73565. The data clearly reveal that in TAD1, the expression level of genes associated with active replication origins (ORI1, ORI2, ORI3) are several folds of that associated with dormant replication origins (ORI4, ORI5) (3 replicates). We note that while the active origin (ORI7) the late replicating TAD2 is not exposed to the domain periphery in the early S phase, its associated gene RCC2 is actively regulated replication timing of RDs $[4,5]$.

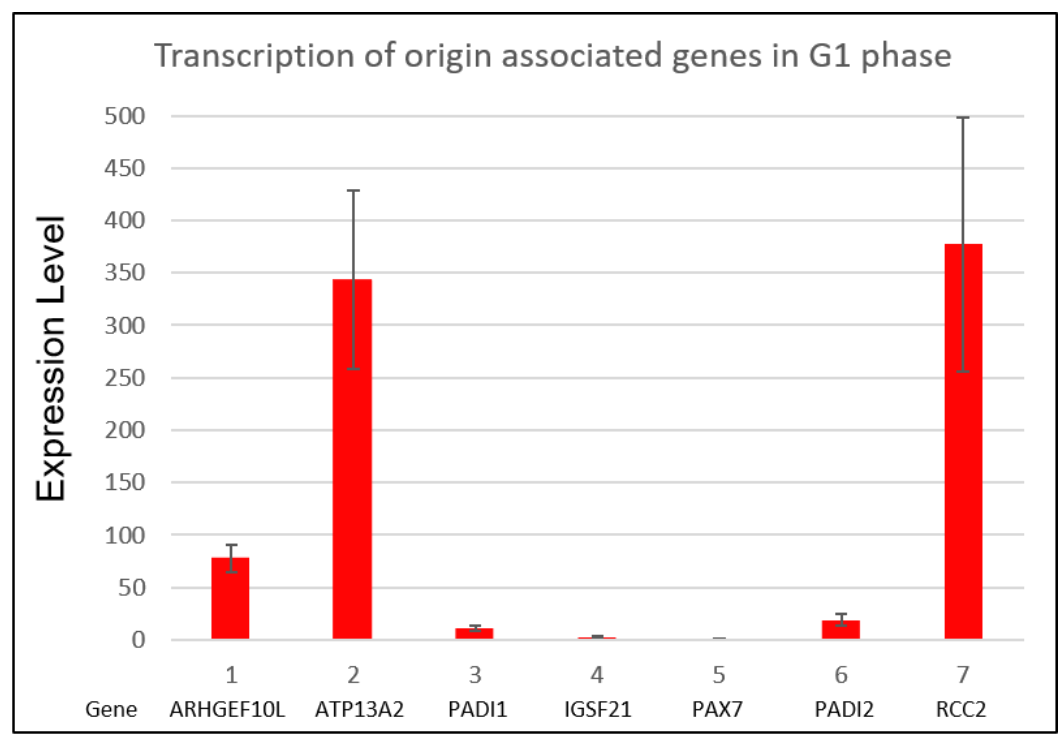




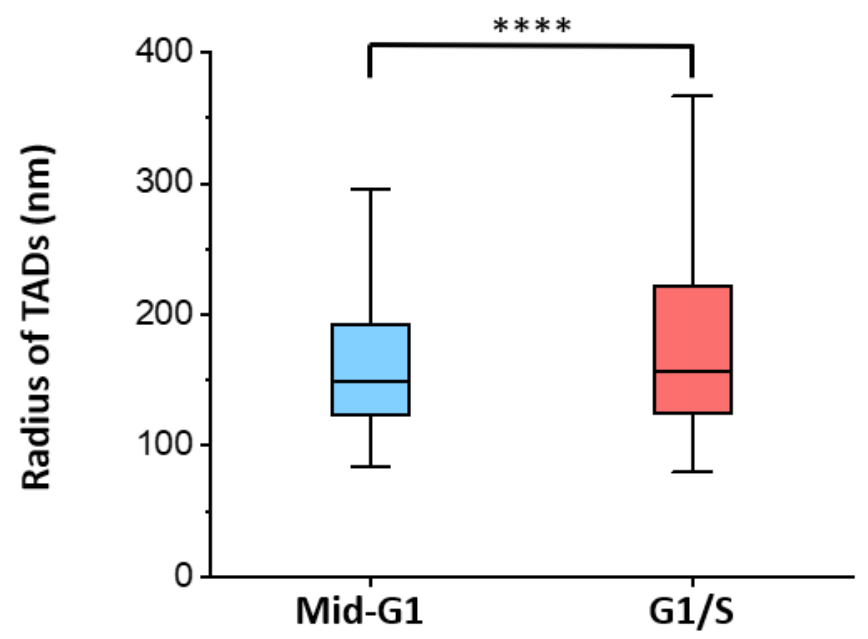

222 Appendix Figure S12. Radii of metabolically labeled TADs in the G1 and G1/S phase.

223 TADs were labeled by EdU for 45 min upon release into the S phase. In the next cell cycle, cells 224 were fixed in the mid-G1 or G1/S phase. For lines and statistics see the description in the legend 225 of Figure $1(\mathrm{n}=10$ cells). 
a

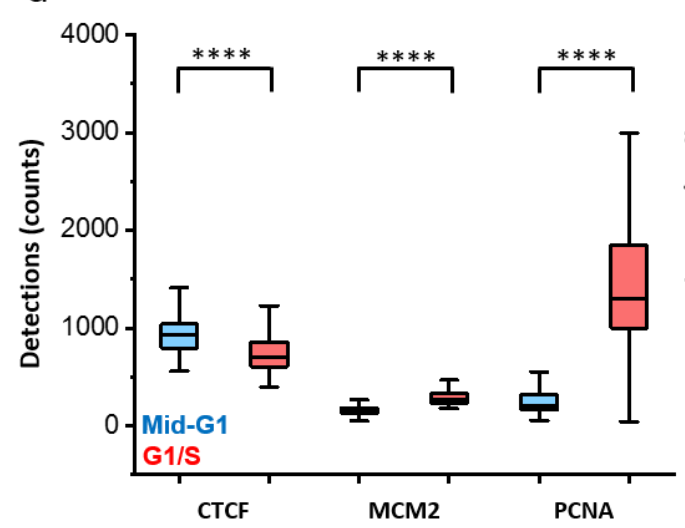

b

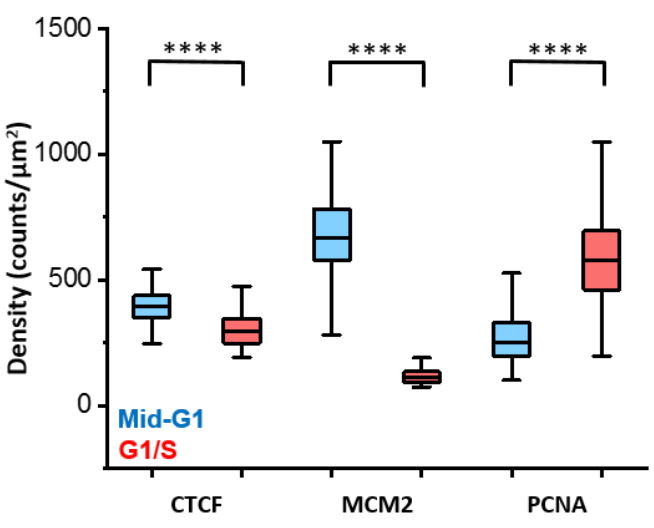

230 Appendix Figure S13. Single molecule detection counts and density of CTCT, MCM2, and 231 PCNA in early replicating TADs in the mid-G1 and G1/S phases. Counts are shown in a and molecule density is shown in $\mathbf{b}$. The reduced number of single-molecule CTCF detections and molecule density in the CTCF foci indicate that CTCF molecules dissociate from chromatin in the G1 phase. The increased number of single-molecule MCM2 detections and decreased molecule density in the MCM2 foci indicate gradual association of MCM2 with chromatin and dislocation from DNA. The increased number of single-molecule PCNA detections and molecule density in the 237 PCNA foci indicate assembly of replication factories in the G1 phase. See details in the main text. 238 For lines and statistics see the description in the legend of Figure $1(\mathrm{n}=10$ cells). 
bioRxiv preprint doi: https://doi.org/10.1101/2020.08.16.252668; this version posted August 17, 2020. The copyright holder for this preprint (which was not certified by peer review) is the author/funder. All rights reserved. No reuse allowed without permission.

Additional File Table 1 Information for TADs and origins.

241

\begin{tabular}{|c|c|c|c|c|c|}
\hline RD & Description & Locus name & $\begin{array}{l}\text { Sequence in } \\
\text { the TAD }\end{array}$ & Sequence in the Genome (hg38) & Length (kb) \\
\hline \multirow{7}{*}{ TAD1 } & $\begin{array}{l}\text { A TAD replicating } \\
\text { in early S phase } \\
\text { with multiple } \\
\text { origins }\end{array}$ & TAD1 & $1-802996$ & 16911932-17714928 & 803 \\
\hline & TAD boundaries & TB & $\begin{array}{c}1-40000 \\
763000-802996\end{array}$ & $\begin{array}{l}16911932-16951772 \\
17674931-17714931\end{array}$ & 40 \\
\hline & $\begin{array}{l}\text { TAD boundary and } \\
\text { active origin }\end{array}$ & ORI1 & 1-19840 & 16911932-16931772 & 20 \\
\hline & \multirow{2}{*}{ Active origins } & ORI2 & $59361-78720$ & 16971293-16990652 & 20 \\
\hline & & ORI3 & $283841-321760$ & $17195773-17233692$ & 38 \\
\hline & \multirow{2}{*}{ Dormant origins } & ORI4 & $195041-215040$ & $17106973-17126972$ & 20 \\
\hline & & ORI5 & $509281-529280$ & $17421213-17441212$ & 20 \\
\hline \multirow{4}{*}{ TAD2 } & $\begin{array}{l}\text { A TAD replicating } \\
\text { in middle } S \text { phase } \\
\text { with multiple } \\
\text { origins }\end{array}$ & TAD2 & $1-1123529$ & $17722716-18846245$ & 1123 \\
\hline & TAD boundaries & TB & $\begin{array}{r}1-40000 \\
1083529- \\
1123529\end{array}$ & $\begin{array}{c}17722716-17762716 \\
1083529-1123529\end{array}$ & 40 \\
\hline & \multirow{2}{*}{$\begin{array}{l}\text { active replication } \\
\text { origin }\end{array}$} & ORI6 & 373281-392960 & $18095997-18115676$ & 20 \\
\hline & & ORI7 & $922000-948000$ & $18644716-18670716$ & 26 \\
\hline
\end{tabular}

